\title{
Supporting Information to: Deconvolution of Luminescence Cross-Talk in High-Throughput Gene Expression Profiling
}

\author{
Marco Mauri*, Stefano Vecchione, and Georg Fritz ${ }^{\dagger}$ \\ LOEWE Center for Synthetic Microbiology and Department of Physics, \\ Philipps-Universität Marburg, Germany
}

\section{Contents}

1 Supporting text 1

1.1 Analytical expression of the bleed-through factor . . . . . . . . . . . . . . . 1

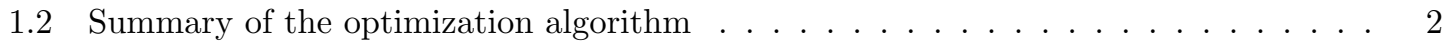

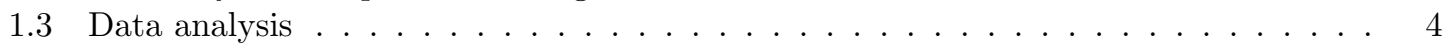

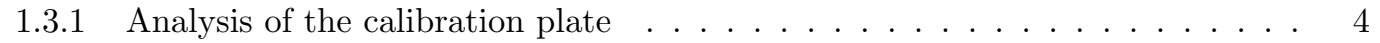

$1.3 .2 \quad$ Analysis of the experiment plate $\ldots \ldots \ldots \ldots . \ldots \ldots$

\begin{tabular}{lll}
\hline 2 & Supporting reference & 15
\end{tabular}

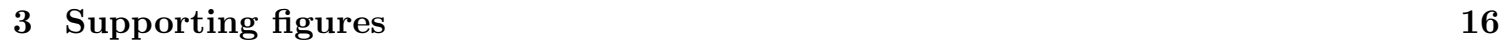

\begin{tabular}{|ll}
\hline & DNA sequence of pSVM-MC_038
\end{tabular}

5 DNA sequence of pSV012

\section{Supporting text}

\subsection{Analytical expression of the bleed-through factor}

Here, we work out the analytical expression Equation (2) of the main text, that expresses the bleedthrough factor as a function of the distance from a single luminescence-emitting well. When the photo-detector is positioned at an offset $h$ from a microplate well $w$, it still receives luminescence incoming from the emitting well $E 5$ under a small angle $\theta$, as shown in Figure $\mathrm{S} 2$. Calling $d$ the distance between neighbouring wells, the detector that is positioned $x$ wells away from $E 5$ detects

${ }^{*}$ Current address: Univ. Grenoble Alpes, Inria, 38000 Grenoble, France

${ }^{\dagger}$ For correspondence: georg.fritz@synmikro.uni-marburg.de 
a background-subtracted intensity $O(t)$ at a time $t$ that is inversely proportional to the square of the distance from the source $l(x)$

$$
O(t, x) \propto \frac{\cos \theta(x)}{l(x)^{2}} .
$$

From trigonometry considerations, the cosine of the angle $\theta$ can be expressed by

$$
\cos \theta(x)=\frac{h}{\sqrt{h^{2}+(x d)^{2}}}
$$

and the distance from the source $l(x)$ by

$$
l(x)=\sqrt{h^{2}+(x d)^{2}} .
$$

We obtain the time-averaged bleed-through factor $B(x)$ by rescaling $O(t, x)$ with respect to the reference intensity measured above the luminescent well $O(t, 0)=1 / h^{2}$ and averaging over the measured time interval $T$

$$
\begin{aligned}
B(x) & \equiv \frac{1}{T} \sum_{t, t^{\prime}=0}^{T} \frac{O\left(t^{\prime}, x\right)}{O(t, 0)}= \\
& =\frac{1}{T} \sum_{t, t^{\prime}=0}^{T} \frac{h}{\sqrt{h^{2}+(x d)^{2}}} \frac{1}{h^{2}+(x d)^{2}} h^{2}= \\
& =\frac{h^{3}}{\left(h^{2}+(x d)^{2}\right)^{3 / 2}}
\end{aligned}
$$

with $l(0)=h$ and $\cos \theta(0)=1$. If rows and columns of the microplates are identified by index $m$ and $n$, respectively, where $m=1, \ldots, 8$ and $n=1, \ldots, 12$, the distance $x$ from the emitting well E5 can be explicitly written as

$$
x=\sqrt{(m-5)^{2}+(n-5)^{2}} .
$$

Substituting $x$, we finally obtain

$$
B_{m, n}=\frac{h^{3}}{\left(h^{2}+d^{2}\left((m-5)^{2}+(n-5)^{2}\right)\right)^{3 / 2}} .
$$

Equation 6 is the theory prediction of the time-averaged bleed-through factor as a function of the distance of the emitting well, as stated from Equation (2) in the main text.

\subsection{Summary of the optimization algorithm}

We give here a brief summary of the optimization algorithm to find the kernel $D$. We first measure the luminescence intensities of the calibration plate $O_{m, n}(t)$ as described in the main text and arrange them by row concatenation into a vector $\overrightarrow{O(t)}$. Then, at every iteration step $k$, we

1. find the bleed-through factor matrix $B(t)$, with entries $B_{m, n}(t)$ given by Equation (1) in the main text

$$
B_{m, n}(t)=\frac{O_{m, n}(t)}{O_{5,5}(t)}
$$


2. time-average $B(t)$ to obtain the time-averaged bleed-through factor matrix $B$ (with entries $B_{m, n}$ ) and the corresponding matrix of standard deviations $\sigma^{B}$ (with entries $\sigma_{m, n}^{B}$ )

3. assemble the matrix $E$ (with entries $E_{i, j}$ ) and the matrix of standard deviations $\sigma^{E}$ (with entries $\sigma_{i, j}^{E}$ ) from $B$ and $\sigma^{B}$, respectively, according to

$$
E_{i, j}= \begin{cases}B_{i-3, j-7}, & \text { if } 4 \leq i \leq 11,8 \leq j \leq 19 \\ B(x), & \text { otherwise, for all }(\mathrm{i}, \mathrm{j}) \text { at distance } x=\sqrt{(i-8)^{2}+(j-12)^{2}} \text { from E5 } \\ z, & \text { otherwise, }\end{cases}
$$

The first line represents the measured time-averaged bleed-through matrix $B . \overline{B(x)}$ in the second line represents the average of the measured values of $B$ that are at a distance $x=$ $\sqrt{(i-8)^{2}+(j-12)^{2}}$ from $E 5$. This result is used to fill entries at the same distance $x$ outside the measured plate. All other entries of the extended matrix $E$ are filled with $z$, computed by averaging intensities over the wells defined as background (A12-H12). A graphical explanation of the composition of the entries of $E$ is given in Figure 95

4. create a matrix $\widetilde{E}$ with entries given by Equation (6) in the main text

$$
\widetilde{E}_{i, j}=E_{i, j}+r_{i, j} \sigma_{i, j}^{E}
$$

where $r_{i, j}$ is a random number generated from a gaussian distribution with mean 0 and standard deviation 1

5. follow the prescription of Equation (3) in the main text, where $E$ is replaced by $\widetilde{E}$, to assemble the kernel $D$ with entries

$$
D_{a, b}=\widetilde{E}_{m-l+8, n-p+12}
$$

where $a=12(m-1)+n, b=12(l-1)+p$ with $m, l=1, \ldots, 8$ and $n, p=1, \ldots, 12$

6. find the vector of deconvolved intensities $\overrightarrow{R(t)}$ by applying Equation (5) in the main text

$$
\overrightarrow{R(t)}=D^{-1} \cdot \overrightarrow{O(t)}
$$

7. after transforming the vector $\overrightarrow{R(t)}$ into a matrix with entries $R_{i, j}(t)$, compute the difference between each $R_{i, j}(t)$ and the instrument sensitivity $s$ (excluding luminescent well $E 5$ ):

$$
Q_{i, j}=R_{i, j}(t)-s
$$

8. if every $Q_{i, j} \leq 0$, iterations stop; otherwise $D$ is stored as $D_{\text {best }}=\prod_{k} D_{k}$, where the product is intended as matrix multiplication and for $k=1, D_{0}$ is the identity matrix

9. apply the acceptance criteria:

- if $R(t)<s$ or $R_{i, j}(t)<O_{i, j}(t)$, the bleed-through has been reduced and $R_{i, j}(t)$ is accepted

- otherwise we set $R_{i, j}(t)=O_{i, j}(t)$ 
10. assume that the luminescence left-overs are the new observed intensities, by setting $O_{i, j}(t)=$ $R_{i, j}(t)$

11. repeat from point 1$)$.

The acceptance criteria ensure that the correction affects the wells where there is still present some residual bleed-through after the deconvolution process.

\subsection{Data analysis}

In this section, we explain in detail how we analyse and correct for luminescence bleed-through the microplate reader data. Although we use a 96 -wells microplate, the process can be extended to any microplate. Rows and columns of the microplates are identified by index $m$ and $n$, respectively, where $m=1, \ldots, 8$ (or by using letters $m=A, \ldots, H$ ) and $n=1, \ldots, 12^{1}$. We prepare the calibration plate by inoculating with a luminescent bacterial strain a single well $(E 5)$, the other wells of the plate with a non-luminescent strain, and proceed by

(a) measuring the luminescence of the plate for 2 hours

(b) adding arabinose (at high concentration, $0.2 \%$ in our case) in $E 5$ to induce maximal luminescence $($ at $\mathrm{t}=0 \mathrm{~h})$

(c) measuring the luminescence of the plate for 6 hours. In general, we record the luminescence signals during the exponential growth phase.

We produce three replicates both of the calibration plate and of the experiment that needs to be bleed-through corrected. The output files of the microplate reader software are processed by our algorithm in MATLAB as described in the following and schematically summarized in Figures 920 and $\mathrm{S} 21$.

\subsubsection{Analysis of the calibration plate}

We follow the scheme of Figures 920.

\section{(1) Raw luminescence $\mathrm{L}$}

We first arrange the raw luminescence data from the microplate reader files into an array $L$ with entries $L_{m, n}(t, c)$, where the indexes $m$ and $n$ indicate row and column of the plate, $t$ the time point (given as machine reading cycle) and $c$ the experimental replicate.

\section{(2) Luminescence background b}

Next, we compute the background $b$ of the luminescence by averaging the values of the luminescence in the wells from A12 to H12 ( 8 wells), before arabinose induction (between $t=-2 \mathrm{~h}$ and $\mathrm{t}=0 \mathrm{~h}$, for

\footnotetext{
${ }^{1}$ Notation: We use the indexes $(m, n)$ to identify the entries of matrices which luminescence values come from direct measurement, such as the bleed-through matrix $B ;(i, j)$ to identify the entries of the matrix $E$, resulting from the extension of $B$ under mathematical prescription; $(a, b)$ to identify the entries of the matrix $D$, and $(\alpha, \beta)$ indicates the position on the plate of the luminescent well. Vectors are denoted by $\vec{V}$.
} 
a total time range $T_{b i}=2 \mathrm{~h}$ ), and over $C$-replicates (in our case three replicates):

$$
b=\frac{1}{8} \frac{1}{T_{b i}} \frac{1}{C} \sum_{m=1}^{8} \sum_{t=1}^{T_{b i}} \sum_{c=1}^{C} L_{m, 12}(t, c)
$$

with variance $\sigma_{b}^{2}$

$$
\sigma_{b}^{2}=\frac{1}{8} \frac{1}{T_{b i}} \frac{1}{C} \sum_{m=1}^{8} \sum_{t=1}^{T_{b i}} \sum_{c=1}^{C}\left(L_{m, 12}(t, c)-b\right)^{2}
$$

and standard deviation $\sigma^{b}=\sqrt{\sigma_{b}^{2}}$.

\section{(3) Instrument sensitivity s}

We also define an instrument sensitivity $s$ as three times the standard deviation of the background

$$
s=3 \sigma^{b}
$$

with the corresponding variance

$$
\sigma_{s}^{2}=9 \sigma_{b}^{2} \sqrt{\frac{2}{8 T_{b i} C-1}}
$$

where we used propagation of uncertainty and variance of variance to obtain $\sigma_{s}^{2}$. Any signal beneath $s$ can not be significantly statistically distinguished from the background value.

\section{(4) Background-subtracted luminescence $\mathrm{O}$}

The background-subtracted luminescence is obtained by subtracting the background from the raw luminescence:

$$
O_{m, n}(t, c)=L_{m, n}(t, c)-b
$$

with the corresponding variance

$$
\sigma_{O}^{2}(t, c)_{m, n}=\sigma_{b}^{2} .
$$

We arrange the standard deviations computed from the variance in the array $\sigma^{O}(t, c)=\sqrt{\sigma_{O}^{2}(t, c)}$.

\section{(5) Replica average of $\mathrm{O}$}

Then, we weight-average the background-subtracted luminescence $O$ over $C$ replicates, such that $O$ has entries

$$
O_{m, n}(t)=\frac{\sum_{c=1}^{C} w_{O}(t, c)_{m, n} O_{m, n}(t, c)}{\sum_{c=1}^{C} w_{O}(t, c)_{m, n}}
$$

with weights $w_{O}(t, c)_{m, n}=1 / \sigma_{O}^{2}(t, c)_{m, n}$ and variance

$$
\sigma_{O}^{2}(t)_{m, n}=\frac{\sum_{c=1}^{C} w_{O}(t, c)_{m, n}\left(O_{m, n}(t, c)-O_{m, n}(t)\right)^{2}}{\sum_{c=1}^{C} w_{O}(t, c)_{m, n}} .
$$

We arrange the standard deviations computed from the variance in the array $\sigma^{O}(t)=\sqrt{\sigma_{O}^{2}(t)}$. 


\section{(6) Bleed-through factor $B$}

The bleed-through factor $B$ in a given well $(m, n)$ at time $t$ is the ratio between the observed background-subtracted luminescence intensities $O$ in such well and in the luminescent well E5:

$$
\begin{aligned}
B_{m, n}(t) & =\frac{O_{m, n}(t)}{O_{5,5}(t)} \\
\sigma_{B}^{2}(t)_{m, n} & =\left(\frac{O_{m, n}(t)}{O_{5,5}(t)}\right)^{2}\left[\left(\frac{\sigma^{O}(t)_{m, n}}{O_{m, n}(t)}\right)^{2}+\left(\frac{\sigma^{O}(t)_{5,5}}{O_{5,5}(t)}\right)^{2}-2 \frac{\operatorname{cov}\left(O_{5,5}(t), O_{m, n}(t)\right)}{O_{m, n}(t) O_{5,5}(t)}\right] .
\end{aligned}
$$

with $\sigma^{B}(t)=\sqrt{\sigma_{B}^{2}(t)}$ defined as the array of the standard deviations.

\section{(7) Selection of time interval to average $B$}

As discussed in main text (Figure 1C), since for a specific well the bleed-through factor is almost constant at any time point after induction, we average its value over a time interval $T$.

\section{(8) Time-averaged bleed-through factor B}

Then, we time-average the bleed-through factor

$$
B_{m, n}=\frac{\sum_{T} w_{B}(t)_{m, n} B_{m, n}(t)}{\sum_{T} w_{B}(t)_{m, n}}
$$

with weights $w_{B}(t)_{m, n}=1 / \sigma_{B}^{2}(t)_{m, n}$ and weighted variance

$$
\sigma_{B, m, n}^{2}=\frac{\sum_{T} w_{B}(t)_{m, n}\left(B_{m, n}(t)-B_{m, n}\right)^{2}}{\sum_{T} w_{B}(t)_{m, n}} .
$$

We arrange the standard deviations into a matrix $\sigma^{B}$. If we assume for simplicity that all weights $w$ are equal, Equation 23 becomes the analytical Equation 4

\section{(9) Extended matrix E}

The observed luminescence intensity above a given well is given by the sum of the true intensity and several bleed-through intensities. The assumption that any luminescent well generates a light patter that resembles the one measured in the case of a single luminescent well corresponds mathematically to convolve the unknown pattern of real intensities $R$ with the time-averaged bleed-through matrix $B$

$$
O(t)=B * R(t)
$$

To perform this convolution with a 96 -well microplate, it is necessary to extend the matrix of the bleed-through factors $B$ to a $15 \times 23$ matrix $E$, as shown in Figure 54 . We extend $B$ according to the following prescription:

$$
E_{i, j}= \begin{cases}B_{i-3, j-7}, & \text { if } 4 \leq i \leq 11,8 \leq j \leq 19 \\ B(x), & \text { otherwise, for all }(\mathrm{i}, \mathrm{j}) \text { at distance } x=\sqrt{(i-8)^{2}+(j-12)^{2}} \text { from } E 5 \\ z, & \text { otherwise }\end{cases}
$$


where $i=1, \ldots, 15$ and $j=1, \ldots, 23$. The first line represents the measured time-averaged bleedthrough matrix $B$ from Equation 23 graphically represented as the coloured central matrix of Figure S5. The second line contains the average of entries of $B$ located at the same number of well-distance $x=\sqrt{(i-8)^{2}+(j-12)^{2}}$ from E5, represented as the magenta area of Figure $\$ 5$. Explicitly

$$
\begin{aligned}
\overline{B_{i, j}} \equiv \overline{B(x)} & =\frac{\sum_{x} w_{B(x), m, n} B_{m, n}}{\sum_{x} w_{B(x), m, n}} \\
w_{B(x), m, n} & =1 / \sigma_{B, m, n}^{2}
\end{aligned}
$$

where indexes $m, n$ refers as before to the wells of the measured matrix. A graphical example of how such entries are computed is given in Figure $\$ 5$ measured values at a fixed distance from $E 5$ are averaged and the result is used to fill the entries at the same distance outside the measured plate. All other entries of the extended matrix $E$ are filled with $z$, computed by averaging intensities over the wells defined as background (A12-H12). This last is the brown area of Figure $\$ 5$. The final outcome of such prescription is given in Figure $\$ 5$ The corresponding variance results

$$
\sigma_{E, i, j}^{2}= \begin{cases}\sigma_{B, i-3, j-7}^{2}, & \text { if } 4 \leq i \leq 11,8 \leq j \leq 19 \\ \sigma_{\bar{B}}^{2}, & \text { otherwise, for all wells at distance x from E5 } \\ \sigma_{z}^{2}, & \text { otherwise }\end{cases}
$$

with

$$
\begin{aligned}
\sigma_{B(x), m, n}^{2} & =\frac{\sum_{x} w_{B(x), m, n}\left(B_{m, n}-\overline{B(x)}\right)^{2}}{\sum_{x} w_{B(x), m, n}} \\
w_{B(x), m, n} & =1 / \sigma_{B, m, n}^{2}
\end{aligned}
$$

where $x$ indicates again the distance from $E 5$ and

$$
\begin{aligned}
z & =\frac{\sum_{m=1}^{8} w_{B, m, 12} B_{m, 12}}{\sum_{m=1}^{8} w_{B, m, 12}} \\
\sigma_{z}^{2} & =\frac{\sum_{m=1}^{8} w_{B, m, 12}\left(B_{m, 12}-z\right)^{2}}{\sum_{m=1}^{8} w_{B, m, 12}} \\
w_{B, m, n} & =1 / \sigma_{B, m, n}^{2} .
\end{aligned}
$$

We arrange the standard deviations of $E$ into a matrix $\sigma^{E}$ with entries $\sigma_{i, j}^{E}=\sqrt{\sigma_{E, i, j}^{2}}$. The array of the observed intensities $O$ is therefore given by the discrete convolution of $E$ and $R$ (Equation 25, with entries

$$
O_{m, n}(t)=\sum_{l=1}^{8} \sum_{p=1}^{12} E_{m-l+8, n-p+12} R_{l, p}(t) .
$$




\section{(10) Kernel D}

In order to write Equation 35 as a matrix product, we first arrange $O$ and $R$ into vectors with 96 entries by row concatenation

$$
\begin{aligned}
& \overrightarrow{O(t)}=\left(O_{1,1}(t), O_{1,2}(t), \ldots, O_{1,12}(t), \ldots, O_{8,1}(t), \ldots, O_{8,12}(t)\right)^{T} \\
& \overrightarrow{R(t)}=\left(R_{1,1}(t), R_{1,2}(t), \ldots, R_{1,12}(t), \ldots, R_{8,1}(t), \ldots, R_{8,12}(t)\right)^{T}
\end{aligned}
$$

and $E$ into a $96 \times 96$ matrix that we called kernel $D$, with entries given by Equation 35 .

$$
D_{a, b}=E_{m-l+8, n-p+12},
$$

where $a=12(m-1)+n, b=8(l-1)+p$ with $m, l=1, \ldots, 8$ and $n, p=1, \ldots, 12 . \quad$ A graphical example of such matrix is given in Figure $\$ 5$. Equation 35 can be rewritten in matrix form as

$$
\overrightarrow{O(t)}=D \cdot \overrightarrow{R(t)}
$$

The variance $\sigma_{D}^{2}$ follows from the same prescription used to build $D$. Figure $\$ 6$ shows a graphical representation of the entries of the kernel $D$. We call the kernel assembled from Equation 26 the average kernel $D$, since it takes into account only the average values of the bleed-through factor $B$. We arrange standard deviations of the kernel into a matrix $\sigma^{D}$ with entries $\sigma_{f, g}^{D}=\sqrt{\sigma_{D, f, g}^{2}}$ where $f=1, \ldots,(M \cdot N), g=1, \ldots,(M \cdot N)$ and in our case $M=8, N=12$.

\section{1 Generalization of $\mathrm{E}$ and $\mathrm{D}$}

More generally, for a plate with $M$ rows and $N$ columns, Equation 35 becomes

$$
O_{m, n}(t)=\sum_{l=1}^{M} \sum_{p=1}^{N} E_{m-l+M, n-p+N} R_{l, p}(t) .
$$

where $E$ is a matrix with dimension $(2 M-1) \times(2 N-1)$. This equation can be rewritten as a matrix product. We first transform $O$ and $R$ into vectors with $M \cdot N$ entries by row concatenation

$$
\begin{aligned}
& \overrightarrow{O(t)}=\left(O_{1}(t), O_{2}(t), \ldots, O_{M \cdot N}(t)\right)^{T} \\
& \overrightarrow{R(t)}=\left(R_{1}(t), R_{2}(t), \ldots, R_{M \cdot N}(t)\right)^{T}
\end{aligned}
$$

where we have re-labelled the entries. Then, if the calibration plate has luminescent well in position $(\alpha, \beta)$, we build $E$ according to

$$
E_{i, j}= \begin{cases}B_{i+\alpha-M, j+\beta-N}, & \text { if } M-\alpha+1 \leq i \leq 2 M-\alpha, N-\beta+1 \leq j \leq 2 N-\beta \\ B(x), & \text { otherwise, for all wells at distance } \mathrm{x} \text { from }(\alpha, \beta) \\ z, & \text { otherwise },\end{cases}
$$

where $\overline{B(x)}$ represents the average of entries of $B$ at the same distance $x=\sqrt{(m-\alpha)^{2}+(n-\beta)^{2}}$ from the luminescent well $(\alpha, \beta)$ and $z$ is computed by averaging intensities over the wells defined 
in $B$ as background. Finally, we obtain $\vec{O}$ from the matrix product

$$
\overrightarrow{O_{N \cdot(m-1)+n}(t)}=\sum_{l=1}^{M} \sum_{p=1}^{N} E_{M-l+m, N-p+n} \overrightarrow{R_{N \cdot(l-1)+p}(t)}
$$

with $m=1, \ldots, M$ and $n=1, \ldots, N$, or

$$
\overrightarrow{O(t)}=D \cdot \overrightarrow{R(t)}
$$

where we have arranged the entries of $E$ into a $(M \cdot N) \times(M \cdot N)$ matrix called kernel $D$ with entries

$$
D_{a, b}=E_{M-l+m, N-p+n}
$$

where $a=N(m-1)+n, b=N(l-1)+p$ with $m, l=1, \ldots, M$ and $n, p=1, \ldots, N$. The process of building the kernel $D$ from $E$ corresponds to the creation of the associated block-Toeplitz matrix.

\subsection{A simple example of $\mathrm{E}$ and $\mathrm{D}$}

We can explicitly work out a simple example with a plate with dimension $(M, N)=(3,4)$ and the luminescent well in $(\alpha, \beta)=(2,3)$ (in red in the following). The measured time-averaged bleed-through matrix $B$ is given by Equation 23

$$
B=\left[\begin{array}{cccc}
B_{1,1} & B_{1,2} & B_{1,3} & B_{1,4} \\
B_{2,1} & B_{2,2} & B_{2,3} & B_{2,4} \\
B_{3,1} & B_{3,2} & B_{3,3} & B_{3,4}
\end{array}\right]
$$

and graphically represented in upper panel of Figure $\$ 7$ The extended matrix $E$ has dimensions $(2 M-1) \times(2 N-1)=5 \times 7$ and is given by

$$
E=\left[\begin{array}{l|llll|ll}
E_{1,1} & E_{1,2} & E_{1,3} & E_{1,4} & E_{1,5} & E_{1,6} & E_{1,7} \\
\hline E_{2,1} & B_{1,1} & B_{1,2} & B_{1,3} & B_{1,4} & E_{2,6} & E_{2,7} \\
E_{3,1} & B_{2,1} & B_{2,2} & B_{2,3} & B_{2,4} & E_{3,6} & E_{3,7} \\
E_{4,1} & B_{3,1} & B_{3,2} & B_{3,3} & B_{3,4} & E_{4,6} & E_{4,7} \\
\hline E_{5,1} & E_{5,2} & E_{5,3} & E_{5,4} & E_{5,5} & E_{5,6} & E_{5,7}
\end{array}\right] .
$$

The entries are computed according to prescription of Equation 43. Such process is explicitly shown in the second row of Figure $\$ 7$. The final outcome is represented in the third row of Figure :77 the central matrix highlighted in red is the measured $B$, surrounded by $\bar{B}$ built by symmetry and in black the background value $z$. The kernel $D$ has dimension $(M \cdot N) \times(M \cdot N)=12 \times 12$ and each 
Toeplitz block has dimension $N \times N=4 \times 4$. Applying Equation 46

$$
D=\left[\begin{array}{llll|llll|llll}
B_{2,3} & B_{2,2} & B_{2,1} & E_{3,1} & B_{1,3} & B_{1,2} & B_{1,1} & E_{2,1} & E_{1,4} & E_{1,3} & E_{1,2} & E_{1,1} \\
B_{2,4} & B_{2,3} & B_{2,2} & B_{2,1} & B_{1,4} & B_{1,3} & B_{1,2} & B_{1,1} & E_{1,5} & E_{1,4} & E_{1,3} & E_{1,2} \\
E_{3,6} & B_{2,4} & B_{2,3} & B_{2,2} & E_{2,6} & B_{1,4} & B_{1,3} & B_{1,2} & E_{1,6} & E_{1,5} & E_{1,4} & E_{1,3} \\
E_{3,7} & E_{3,6} & B_{2,4} & B_{2,3} & E_{2,7} & E_{2,6} & B_{1,4} & B_{1,3} & E_{1,7} & E_{1,6} & E_{1,5} & E_{1,4} \\
\hline B_{3,3} & B_{3,2} & B_{3,1} & E_{4,1} & B_{2,3} & B_{2,2} & B_{2,1} & E_{3,1} & B_{1,3} & B_{1,2} & B_{1,1} & E_{2,1} \\
B_{3,4} & B_{3,3} & B_{3,2} & B_{3,1} & B_{2,4} & B_{2,3} & B_{2,2} & B_{2,1} & B_{1,4} & B_{1,3} & B_{1,2} & B_{1,1} \\
E_{4,6} & B_{3,4} & E_{4,6} & B_{3,2} & E_{3,6} & B_{2,4} & B_{2,3} & B_{2,2} & E_{2,6} & B_{1,4} & B_{1,3} & B_{1,2} \\
E_{4,7} & E_{4,6} & B_{3,4} & B_{3,3} & E_{3,7} & E_{3,6} & B_{2,4} & B_{2,3} & E_{2,7} & E_{2,6} & B_{1,4} & B_{1,3} \\
\hline E_{5,4} & E_{5,3} & E_{5,2} & E_{5,1} & B_{3,3} & B_{3,2} & B_{3,1} & E_{4,1} & B_{2,3} & B_{2,2} & B_{2,1} & E_{3,1} \\
E_{5,5} & E_{5,4} & E_{5,3} & E_{5,2} & B_{3,4} & B_{3,3} & B_{3,2} & B_{3,1} & B_{2,4} & B_{2,3} & B_{2,2} & B_{2,1} \\
E_{5,6} & E_{5,5} & E_{5,6} & E_{5,3} & E_{4,6} & B_{3,4} & B_{3,3} & B_{3,2} & E_{3,6} & B_{2,4} & B_{2,3} & B_{2,2} \\
E_{5,7} & E_{5,6} & E_{5,5} & E_{5,4} & E_{4,7} & E_{4,6} & B_{3,4} & B_{3,3} & E_{3,7} & E_{3,6} & B_{2,4} & B_{2,3}
\end{array}\right]
$$

that is graphically represented in last row of Figure $\$ 7$ The vector of the observed intensities $\overrightarrow{O(t)}$ and expected corrected intensities $\overrightarrow{R(t)}$ are given by row concatenation

$$
\begin{aligned}
& \overrightarrow{O(t)}=\left(O_{1,1}, O_{1,2}, O_{1,3}, O_{1,4}, O_{2,1}, O_{2,2}, O_{2,3}, O_{2,4}, O_{3,1}, O_{3,2}, O_{3,3}, O_{3,4}\right)(t)^{T} \\
& \overrightarrow{R(t)}=\left(R_{1,1}, R_{1,2}, R_{1,3}, R_{1,4}, R_{2,1}, R_{2,2}, R_{2,3}, R_{2,4}, R_{3,1}, R_{3,2}, R_{3,3}, R_{3,4}\right)(t)^{T} .
\end{aligned}
$$

The final convolution therefore results $\overrightarrow{O(t)}=D \cdot \overrightarrow{R(t)}$.

\section{3 A general example of $\mathrm{E}$ and $\mathrm{D}$}

Given a $B$ matrix with dimension $M \times N$ and luminescent well in $(\alpha, \beta)$

$$
B=\left[\begin{array}{cccccc}
B_{1,1} & B_{1,2} & \ldots & \ldots & \ldots & B_{1, N} \\
\ldots \ldots & \ldots \ldots & \ldots & \ldots & \ldots & \ldots \ldots \\
B_{\alpha, 1} & B_{\alpha, 2} & \ldots & B_{\alpha, \beta} & \ldots & B_{\alpha, N} \\
\ldots \ldots & \ldots \ldots & \ldots & \ldots & \ldots & \ldots \ldots \\
B_{M, 1} & B_{M, 2} & \ldots & \ldots & \ldots & B_{M, N}
\end{array}\right]
$$

the extended matrix $E$ with dimension $(2 M-1) \times(2 N-1)$ reads

$E=\left[\begin{array}{ccc|ccc|ccc}E_{1,1} & \ldots & \ldots & \ldots & \ldots & \ldots & \ldots & \ldots & E_{1,2 N-1} \\ \vdots & \vdots & \vdots & \vdots & \vdots & \vdots & \vdots & \vdots & \vdots \\ \hline E_{M-\alpha+1,1} & \ldots & E_{M-\alpha+1, n-\beta} & B_{1,1} & \ldots & B_{1, N} & E_{M-\alpha+1,2 N-\beta+1} & \ldots & E_{M-\alpha+1,2 N-1} \\ \vdots & \vdots & \vdots & \vdots & E_{M, N} & \vdots & \vdots & \vdots & \vdots \\ E_{2 M-\alpha, 1} & \ldots & E_{2 M-\alpha, n-\beta} & B_{M, 1} & \ldots & B_{M, N} & E_{2 M-\alpha, 2 N-\beta+1} & \ldots & E_{2 M-\alpha, 2 N-1} \\ \hline \vdots & \vdots & \vdots & \vdots & \vdots & \vdots & \vdots & \vdots & \vdots \\ E_{2 M-1,1} & \ldots & \ldots & \ldots & \ldots & \ldots & \ldots & \ldots & E_{2 M-1,2 N-1}\end{array}\right]$


where $E_{M, N} \equiv B_{\alpha, \beta} . D$ is then a block-Toeplitz matrix with dimension $(M \cdot N) \times(M \cdot N)$

$D=\left[\begin{array}{c|c|c|c|c}T\left(E_{M, N}\right) & T\left(E_{M-1, N}\right) & \ldots & \ldots & T\left(E_{1, N}\right) \\ \hline T\left(E_{M+1, N}\right) & T\left(E_{M, N}\right) & T\left(E_{M-1, N}\right) & \ddots & \ddots \\ \hline T\left(E_{M+2, N}\right) & T\left(E_{M+1, N}\right) & T\left(E_{M, N}\right) & \ddots & \ddots \\ \hline \vdots & \ddots & \ddots & \ddots & \ddots \\ \hline T\left(E_{2 M-1, N}\right) & \ddots & \ddots & \ddots & T\left(E_{M, N}\right)\end{array}\right]$

where each block $T\left(E_{x, y}\right)$ is again a Toeplitz matrix

$$
T\left(E_{x, y}\right)=\left[\begin{array}{ccccc}
E_{x, y} & E_{x, y-1} & \ldots & \ldots & E_{x, 1} \\
E_{x, y+1} & E_{x, y} & E_{x, y-1} & \ddots & \ddots \\
E_{x, y+2} & E_{x, y+1} & E_{x, y} & \ddots & \ddots \\
\vdots & \ddots & \ddots & \ddots & \ddots \\
E_{x, 2 N-1} & \ddots & \ddots & \ddots & E_{x, y}
\end{array}\right] .
$$

\section{(11) Best kernel D by iteration}

To take into account uncertainties in the estimation of the bleed-through factors and time fluctuations of luminescence, we replace the matrix $E$ of the average bleed-through factors introduced by Equation 26 by a matrix with entries

$$
\widetilde{E_{i, j}}=E_{i, j}+r_{i, j} \sigma_{i, j}^{E}
$$

where $\sigma^{E}$ and $r$ are matrices which contain the standard deviations of the time-averaged bleedthrough factors (obtained by taking the square root of the entries of Equation 29 ) and the number of standard deviations added to this last, respectively. The variance of $\widetilde{E}$ is given by

$$
\sigma_{\widetilde{E}}^{2}=\sigma_{E}^{2}+r^{2} \sigma_{E}^{2} \sqrt{\frac{2}{T \cdot C-1}}+2 r \cdot \operatorname{cov}\left(E, \sigma^{E}\right),
$$

where the second term is the standard deviation of the standard deviation of $E$, with $C$ the number of replicates and $T$ the time range over which the bleed-through factor $B$ has been averaged. From $\widetilde{E}$, we can compute the kernel $D$ via Equation 38 . We have to look for that $r$ that minimizes the difference between the spurious luminescence signal $R$ after correction by deconvolution and the instrument sensitivity value $s$. Since such minimization is not computationally feasible in a single step, we approach the solution by iteration. The idea is to generate at each iteration a random kernel $D$ and accept or reject it according to minimization criteria. The deconvolved plate is then used as luminescence input for the following iteration. The process is repeated till when there is no luminescence residual above the instrument sensitivity. 


\section{(11). 1 Random kernel D}

We first generate a random $r$ with entries according to a normal distribution $\mathcal{N}(0,1)$, with average 0 and standard deviation 1 . The kernel obtain in such a way, is called random kernel $D^{\text {rnd }}$. An equivalent solution is obtained by directly generating a random kernel $D^{\text {rnd }}$ according to a normal distribution $\mathcal{N}$ with mean the corresponding entries of the average kernel $D$ and standard deviation $\sigma^{D}$ :

$$
D^{\text {rnd }}=\mathcal{N}\left(D, \sigma^{D}\right)
$$

and use such matrix to deconvolve $O$ and find $R$. The matrix of standard deviation $\sigma^{D \text { rnd }}$ is obtained by uncertainty propagation. To keep track of the iteration cycle, we add an iteration index $(k)$ to the notation $\left(D_{(k)}^{\text {rnd }}\right.$ for the kernel array and $R(t)_{(k)}$ for the real intensities array).

\section{Self-correction of the calibration plate}

At each iteration, we substitute the array of real intensities $R$ containing the bleed-through left-overs to the array of observed intensities: $\overrightarrow{O(t)} \equiv \overrightarrow{R(t)_{(k)}}$. Thus, by inverting Equation 39

$$
\overrightarrow{R(t)_{(k+1)}}=\left(D_{(k)}^{\mathrm{rnd}}\right)^{-1} \cdot \overrightarrow{R(t)_{(k)}} .
$$

The propagation of uncertainties during matrix inversion process is discussed in Lefebvre et al. [1] and gives a variance

$$
\sigma_{D^{-1}}^{2}=\left(D^{-1}\right)^{2} \cdot \sigma_{D}^{2} \cdot\left(D^{-1}\right)^{2}
$$

that leads to a variance for $R$ :

$$
\sigma_{\vec{R}}^{2}(t)=\sigma_{D^{-1}}^{2} \cdot \overrightarrow{O(t}^{2}+\left(D^{-1}\right)^{2} \cdot \sigma_{\vec{O}}^{2}
$$

where we assumed the covariance between $O$ and $D$ to be negligible. Square operation is here intended as square of each matrix entry (in MATLAB notation $x .^{\wedge} 2$ ). We can re-arrange $\vec{R}$ into an array $R$ by inverting the row concatenation process.

\section{Bleed-through left-over Q}

After deconvolution, a perfect self-correction of the calibration plate should produce a plate that at any time point has only a single luminescent signal in $E 5$ and all other wells have luminescence value under the instrument sensitivity $s$. We call $I$ the array resulting from such ideal correction. $I$ has entries

$$
I_{m, n}(t)=\left\{\begin{array}{ll}
O_{5,5}(t), & m=5, n=5 \\
s, & \text { otherwise }
\end{array} .\right.
$$

The best correction is achieved after $(K)$ iterations, when there are no more $Q$ left-overs:

$$
Q=\sum_{m=1}^{M} \sum_{N=1}^{N} \sum_{t=1}^{T}\left(R_{m, n}(t)_{(k+1)}-I_{m, n}(t)\right) \equiv 0 .
$$

On the contrary, if after an iteration we still have some left-overs $(Q>0)$, we proceed by applying the acceptance criteria to the left-overs intensity array $R_{(k+1)}$. 


\section{(14) Acceptance criteria}

We apply the acceptance criteria to each entry of the deconvolved $R_{(k+1)}$ independently. If a single entry of $R_{(k+1)}$ has value that is

a) smaller than the previous iteration $R_{m, n,(k+1)}(t) \leq R_{m, n,(k)}(t)$, we accept and store the new value since the bleed-through has been reduced

b) larger than the previous iteration $R_{m, n,(k+1)}(t)>R_{m, n,(k)}(t)$, we reject the move by setting back $R_{m, n,(k+1)}(t)=R_{m, n,(k)}(t)$.

Standard deviation follows the fate of the corresponding entry of $R$. Theoretically, at any time point the bleed-through pattern depends simultaneously on the luminescence of all wells, but here, to guide the convergence of the minimization algorithm, we accept or reject each entry of $R$ separately. This is allowed only if each random iteration perturbs minimally the mean value of the bleed-through factors. We ensure this condition by using instead of $r$ in Equation 47 a much smaller value $a \cdot r$, with $a \ll 1$ (in our case $a=1 / 10$ ).

\section{(15) Update and iterate}

If after $(k)$ iterations there still is some bleed-through $(Q>0)$, we imagine the pattern of deconvolved luminescence $R_{(k+1)}$ as the new observed luminescence $O\left(O \equiv R_{(k+1)}\right)$, update the iteration index $(k)$ and repeat the procedure from point 6 .

\section{(16) Best kernel D}

The best correction is achieved at iteration $(K)$ when, after deconvolution, all left-overs are zero at any time point $(Q=0)$. From Equation 50 , it follows that over the iterations

$$
\begin{array}{rlrl}
\overrightarrow{O(t)} & =D_{(1)}^{\mathrm{rnd}} \overrightarrow{R(t)_{(2)}(t)} & & \text { for } \mathrm{k}=1 \\
\overrightarrow{R_{(2)}(t)} & =D_{(2)}^{\mathrm{rnd}} \overrightarrow{R_{(3)}(t)} & & \text { for } \mathrm{k}=2 \\
\vdots & & \\
\overrightarrow{R_{(K)}(t)} & =D_{(K)}^{\mathrm{rnd}} \overrightarrow{I(t)} & & \text { for } \mathrm{k}=\mathrm{K}
\end{array}
$$

where $\overrightarrow{I(t)}$ is the identity matrix transformed into vector by row concatenation. Therefore

$$
\overrightarrow{O(t)}=\prod_{k=1}^{K} D_{(k)}^{\mathrm{rnd}} \overrightarrow{I(t)} \equiv D^{\text {best }} \overrightarrow{I(t)}
$$

where we define the best kernel via the product of the iteration kernels

$$
D_{\text {best }} \equiv \prod_{k=1}^{K} D_{(k)}^{\text {rnd }} .
$$

The matrices $\sigma_{(k)}^{R}(t)$ and $\sigma_{\text {best }}^{D}$ containing the standard deviations are obtained from Equation 52 and Equation 48, respectively. 


\subsubsection{Analysis of the experiment plate}

We follow the scheme of Figures $\mathrm{S21}$.

\section{Raw luminescence $L$ of the experiment}

Next, we measure the luminescence on the plate that needs to be bleed-through corrected. For simplicity we refer to it as the test plate. We arrange the raw luminescence data into an array $L^{\exp }$ with entries $L_{m, n}^{\exp }(t, c)$, where $m$ and $n$ indicate row and column of the plate, $t$ the time point and $c$ the experiment replica.

\section{(18) Background-subtracted luminescence $O$ of the experiment}

Due to the luminescence bleed-through, the background can not be obtained from the test plate. Instead, we use the cross-talk-free background $b$ from the calibration plate, given by Equation 13 For such reason, it is better if the two measurements are performed under the same conditions and with the same machine setup. The variance of the background is obtained from Equation 14 . After subtracting the background from the raw luminescence, we obtain the array of backgroundsubtracted luminescence $O^{\exp }(t, c)$ with corresponding array of standard deviations $\sigma^{O \exp }(t, c)$.

\section{Bleed-through correction $R$ of the experiment}

The array of observed luminescence is given by the product of the kernel $D_{\text {best }}$ (Equation 60) and the array of unknown "real" intensities $R$ :

$$
\overrightarrow{O^{\exp }(t, c)}=D_{\text {best }} \cdot \overrightarrow{R^{\exp }(t, c)},
$$

where $O$ and $R$ are transformed into vector by row concatenation. We achieve the most probable arrangement of real intensities by inverting the previous equation

$$
\overrightarrow{R^{\exp }(t, c)}=\left(D_{\text {best }}\right)^{-1} \cdot \overrightarrow{O^{\exp }(t, c)} .
$$

Then, we transform back $\overrightarrow{R^{\exp }(t, c)}$ and $\overrightarrow{O^{\exp }(t, c)}$ into arrays $R_{m, n}^{\exp }(t, c)$ and $O_{m, n}^{\exp }(t, c)$, respectively by inverting the row-concatenation process. The array of standard deviation is obtained again by uncertainty propagation as described by Equation 52 .

\section{Replica average of the experiment}

Finally, we average the luminescence over several replicates. The replica-averaged $R^{\exp }(t)$ has entries

$$
R_{m, n}^{\exp }(t)=\frac{\sum_{c=1}^{C} w_{R}^{\exp }(t, c)_{m, n} R_{m, n}^{\exp }(t, c)}{\sum_{c=1}^{C} w_{R}^{\exp }(t, c)_{m, n}}
$$

with weights $w_{R}^{\exp }(t, c)_{m, n}=1 / \sigma_{R}^{\exp 2}(t, c)_{m, n}$ and variance

$$
\sigma_{R}^{\exp 2}(t)_{m, n}=\frac{\sum_{c=1}^{C} w_{R}^{\exp }(t, c)_{m, n}\left(R_{m, n}^{\exp }(t, c)-R_{m, n}^{\exp }(t)\right)^{2}}{\sum_{c=1}^{C} w_{R}^{\exp }(t, c)_{m, n}} .
$$


where $m=1, \ldots, 8, n=1, \ldots, 12$ and $t=1, \ldots, T$. Standard deviations are arranged in an array $\sigma^{R \exp }(t)$, with entries $\sigma_{m, n}^{R \exp }(t)$.

\section{Supporting reference}

[1] Lefebvre M, Keeler RK, Sobie R, and White J. Propagation of errors for matrix inversion. arXiv, 1999. 


\section{Supporting figures}

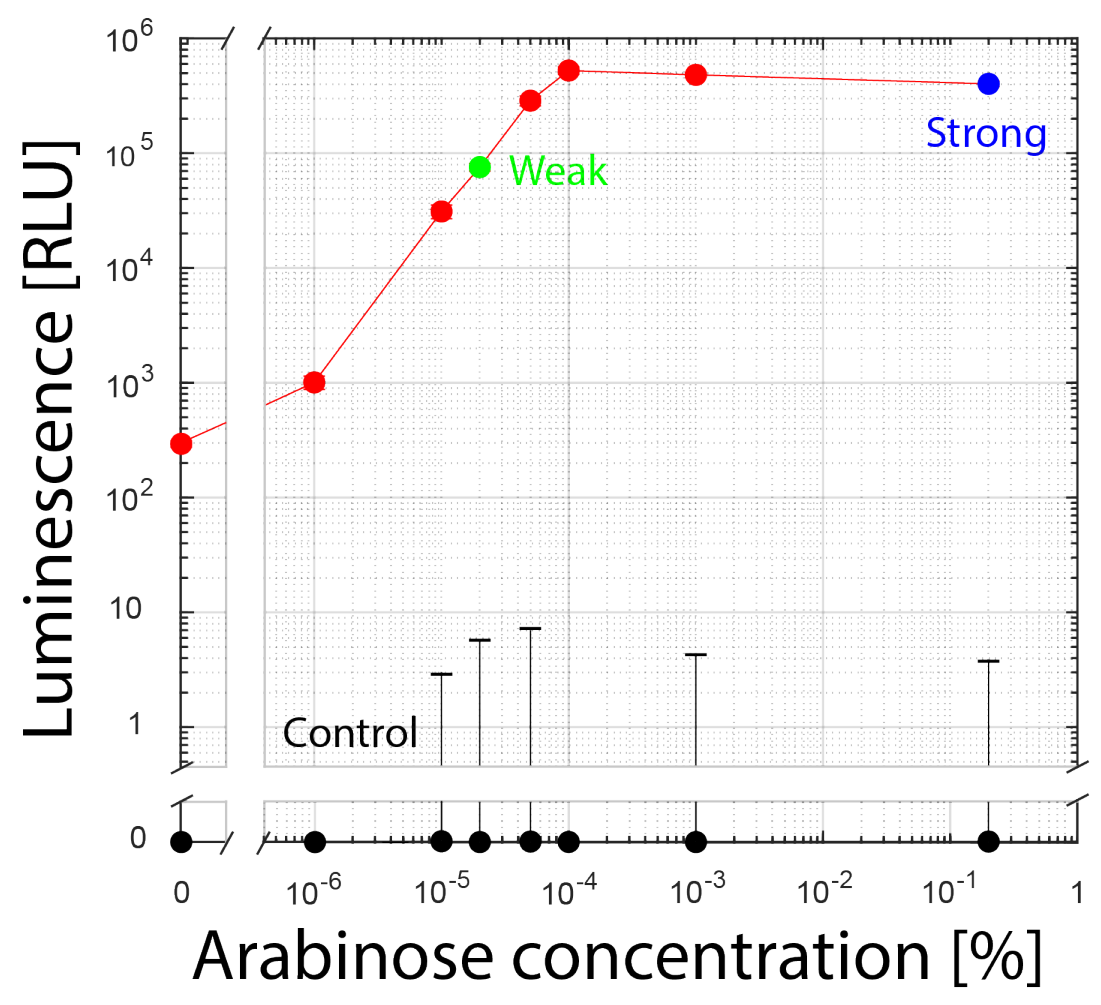

Figure S1: Dose-response plot for the E. coli strain GFC0153: luminescence intensity as function of arabinose concentration for three independent replicates. Luminescence intensities used to assemble the calibration plates with high and low luminescence are indicated as blue and green points, respectively. The plot is obtained from the data of Figure 3E, $3 \mathrm{~F}$ in the main text at 270 minutes after arabinose induction. In black, the intensities of the non-luminescent E. coli strain SV01, used as control, with which is filled the calibration plates (excluding well E5). 


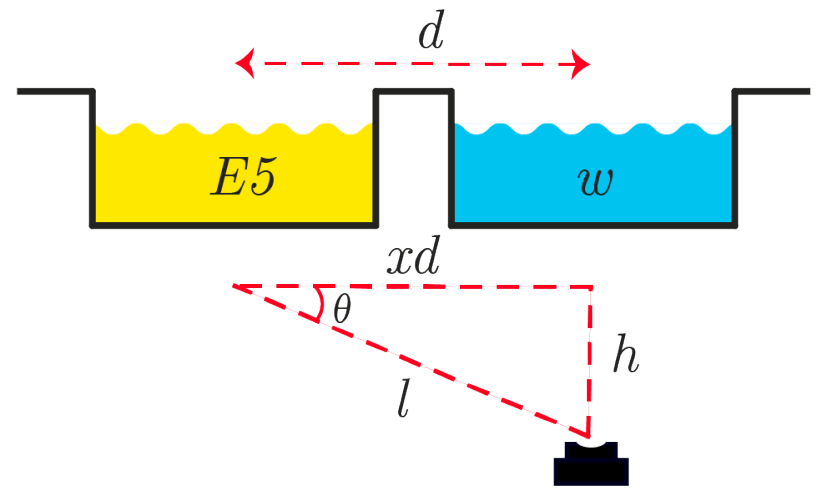

Figure S2: Representation of the microplate reader wells and of the detector. A photo-detector positioned at an offset $h$ from a microplate well $w$ receives luminescence incoming from the emitting well $E 5$ under an angle $\theta$. If $d$ is the distance between neighbouring wells, $x d$ and $l$ are the distances between $E 5$ and $w$ and $E 5$ and the detector, respectively. 

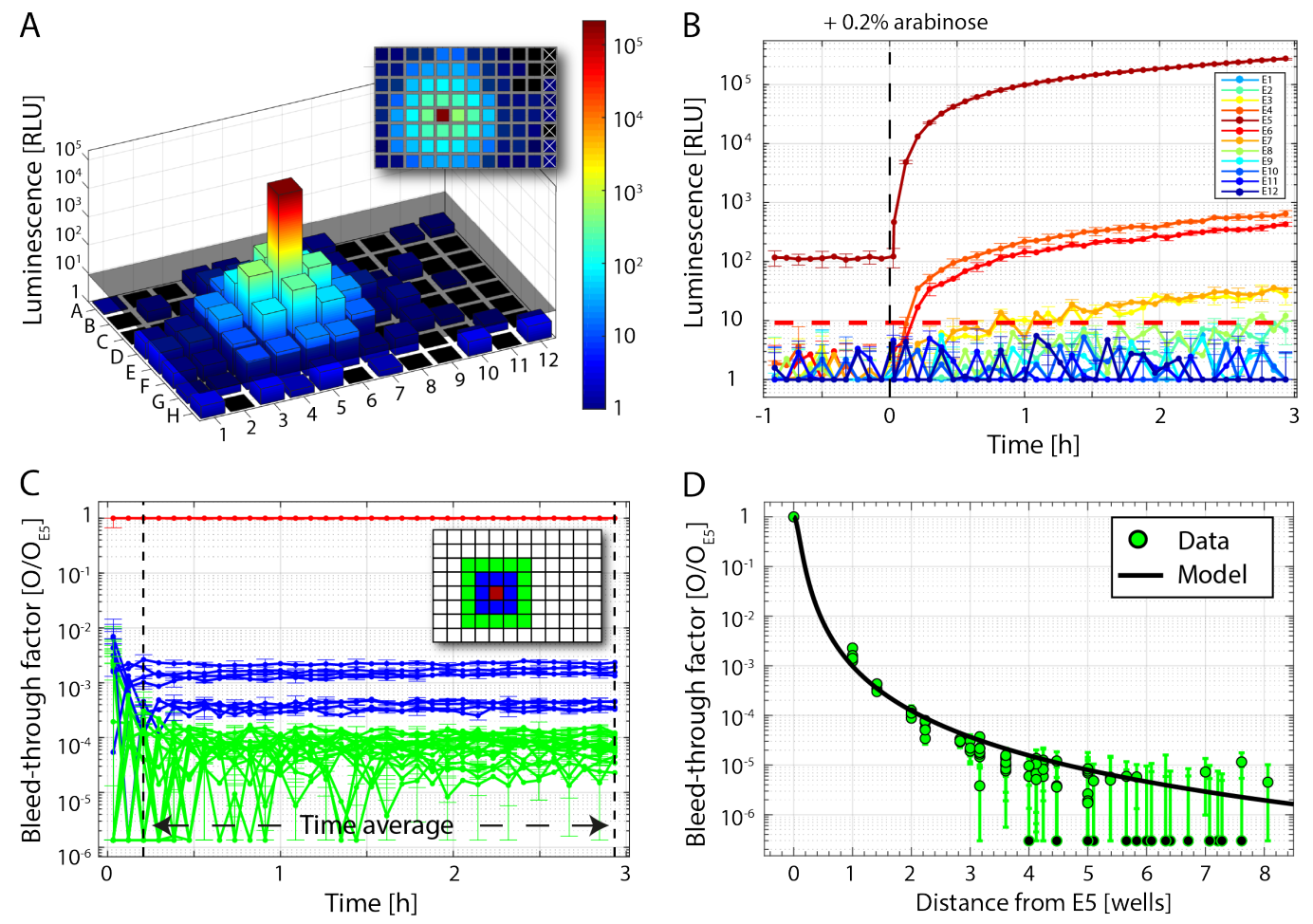

Figure S3: Quantification of luminescence bleed-through in the VICTOR plate reader. (A) The calibration plate was prepared with a single highly luminescent spot in the well E5. All data are background-subtracted and averaged over three experimental replicates. The background was obtained by averaging the signal of the wells indicated by the white crosses in the inset over the time before addition of arabinose (induction time). The 3D-plot shows the observed luminescence signal at 140 minutes after the induction. (B) Time evolution of the observed luminescence signal of the E-row. The red line represents the instrument sensitivity value, defined as three times the standard deviation of the background value. (C) Luminescence bleed-through factor of the two shells closest to $E 5$, in blue and green. Values were obtained dividing the observed signal from a specific well by the signal recorded in $E 5$. The dashed lines indicate the time range over which the bleed-through was averaged to obtain in (D) the bleed-through factor as a function of the distance from E5 (green dots). The black solid line is the parameter free prediction of the bleed-through given by Equation 6 . 


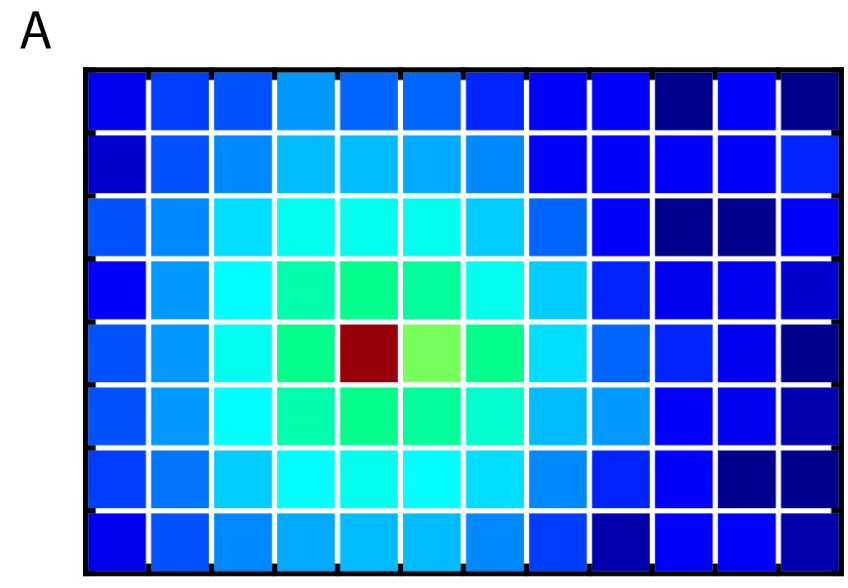

$\operatorname{Max}$
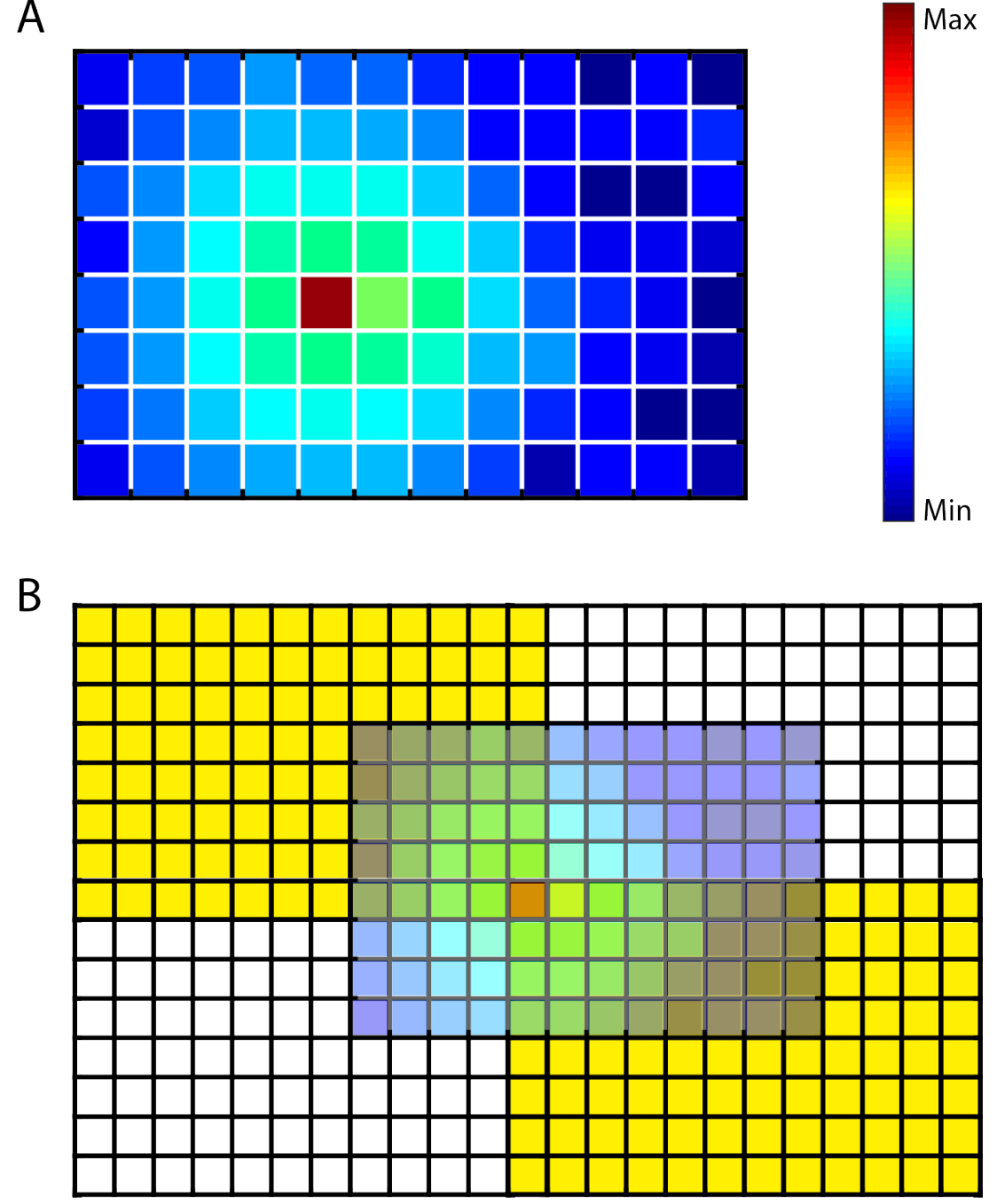

Figure S4: (A) Representation of a bleed-through matrix $B$ generated by measuring the bleedthrough pattern produced in a plate with a single luminescent well E5. (B) To convolve the bleed-through matrix $B$ with the array of real intensities $R$ (in yellow), we enlarge $B$ (in color) to an extended bleed-through matrix $E$ (black grid). 


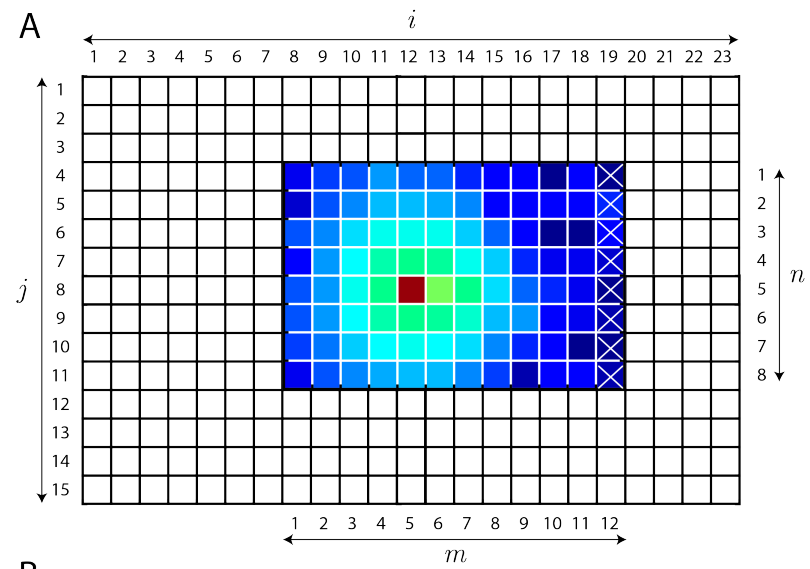

B

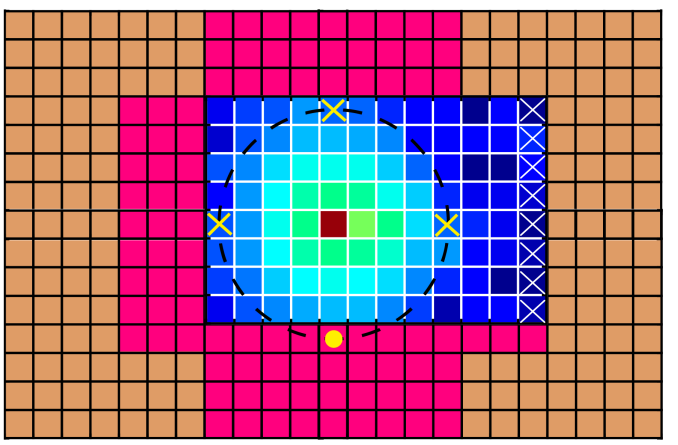

C

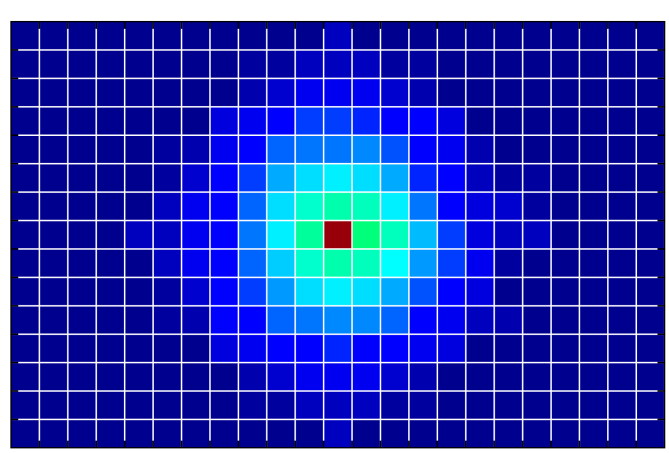

Figure S5: Graphical representation of the entries of an extended matrix $E$ generated by a calibration plate with luminescent well in E5. The central part is occupied by the measured bleed-through factor $B$ (in colors, panel $\mathrm{A}$ ), the magenta area is filled by symmetry with $\overline{B(x)}$ and the brown area with the value $z$ (in panel $\mathrm{B}$ ). The entries of $E$ that we called $\bar{B}$ fall in the magenta area. They are computed by averaging the measured values of $B$ at a same distance $x$ from the luminescent well $E 5$ (see Equation 26). For example, when $x=4$, we average the three measured values in the wells with yellow crosses to obtain the value represented by the yellow dot, that lies outside the measured matrix. The entries of $E$ where such symmetry construction is not possible fall in the brown area. They are given by the value $z$ as indicated in the third line of Equation 26 and computed by averaging the values of $B$ in the wells indicated by the white crosses. Panel $\mathrm{C}$ shows the final result. 


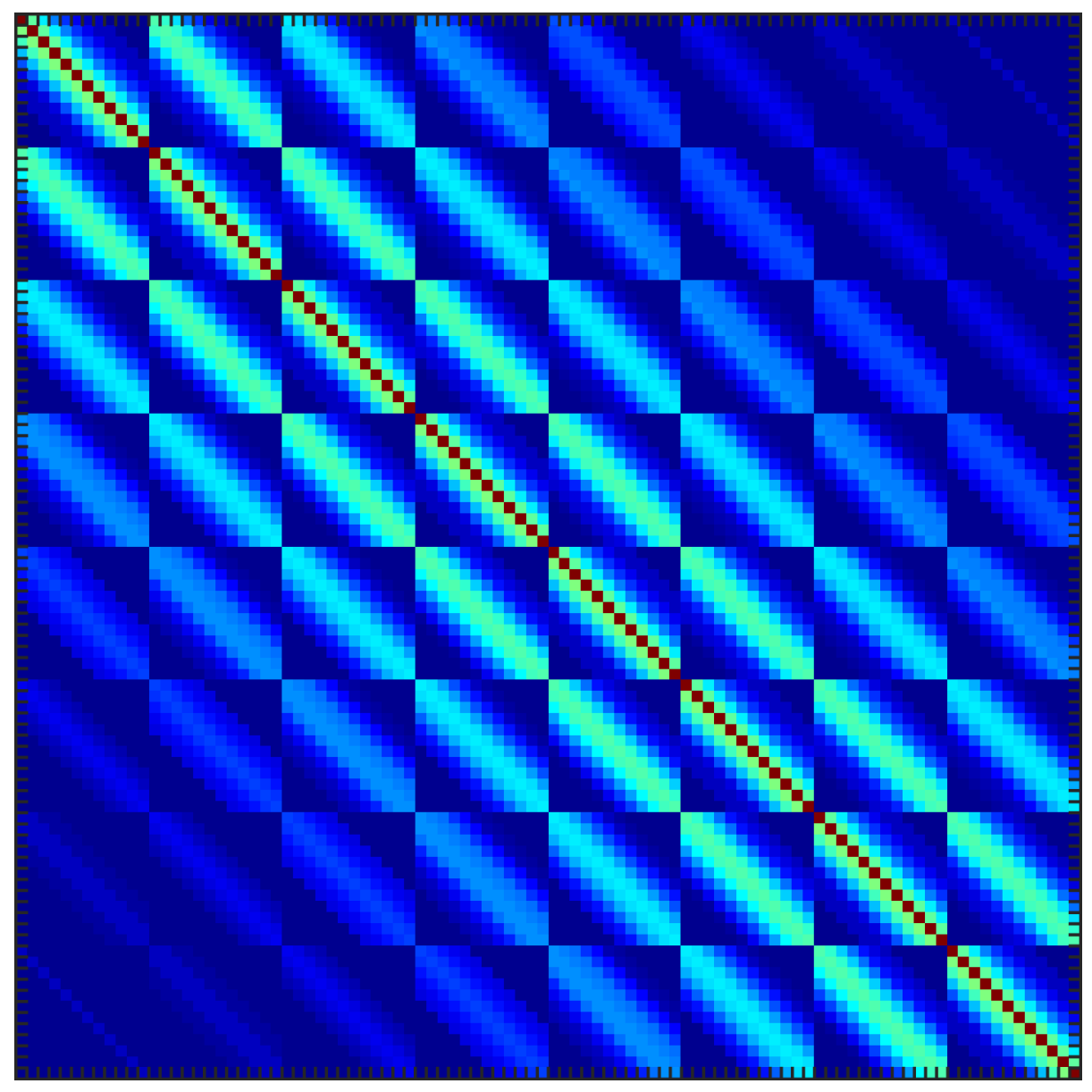

Figure S6: Graphical representation of the entries of the kernel $D$ generated by the extended matrix $E$ of Figure 95 . Each pixel corresponds to an entry. 


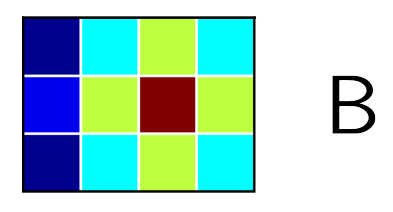

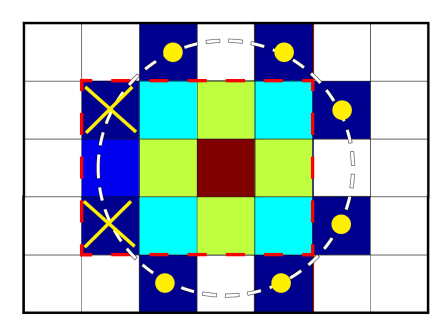

$\overline{\mathrm{B}(\mathrm{x}=2.2)}$

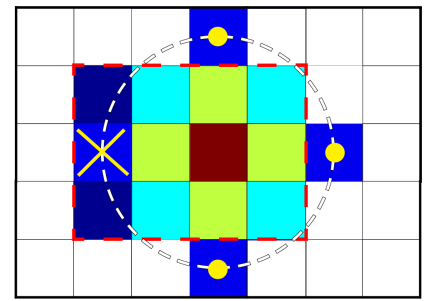

$\overline{\mathrm{B}(\mathrm{x}=2)}$
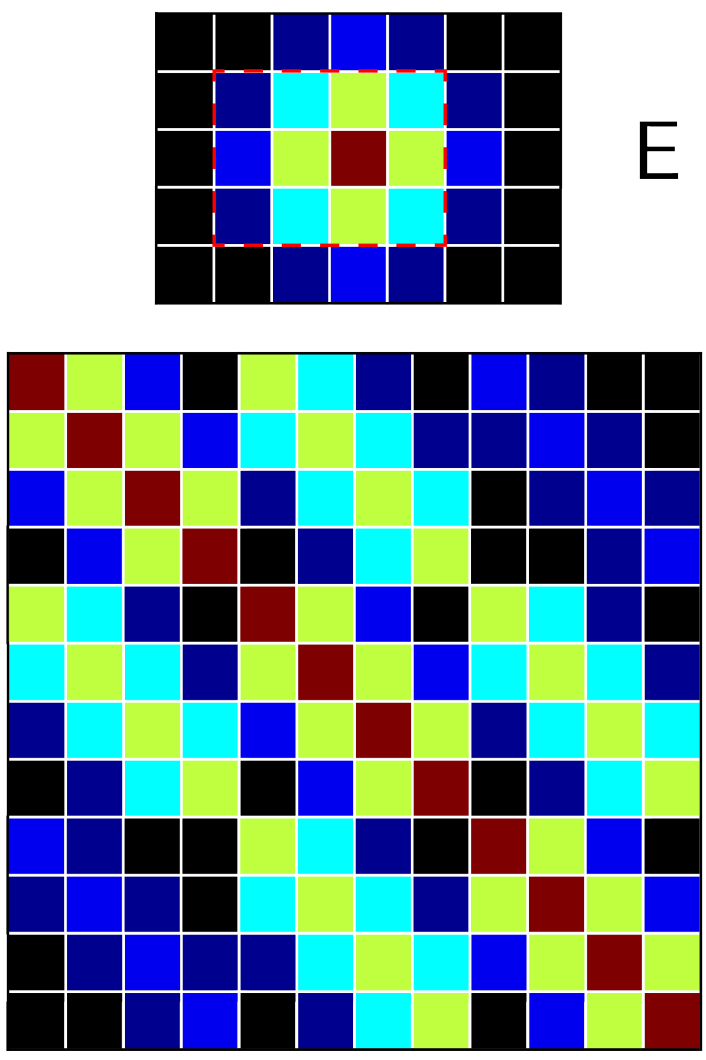

Figure S7: Graphical representation of the example given in section 10.2. The first row shows a measured time-averaged bleed-through matrix $B$. The following rows show step-by-step how we extended the matrix to obtain $E$ : we averaged the measured values of $B$ at a same distance $x$ from the luminescent well, first for $x=\sqrt{5} \simeq 2.2$ and then for $x=2$. Yellow crosses show the measured values. The results are used to fill the entries of $E$ for that specific distance $x$ outside the measured matrix, indicated by yellow dots. We highlight in red the measured values of $B$. The remaining entries of $E$ are filled with the background value $z$, in black. The last row shows the corresponding kernel $D$, computed following Equation 46 . 

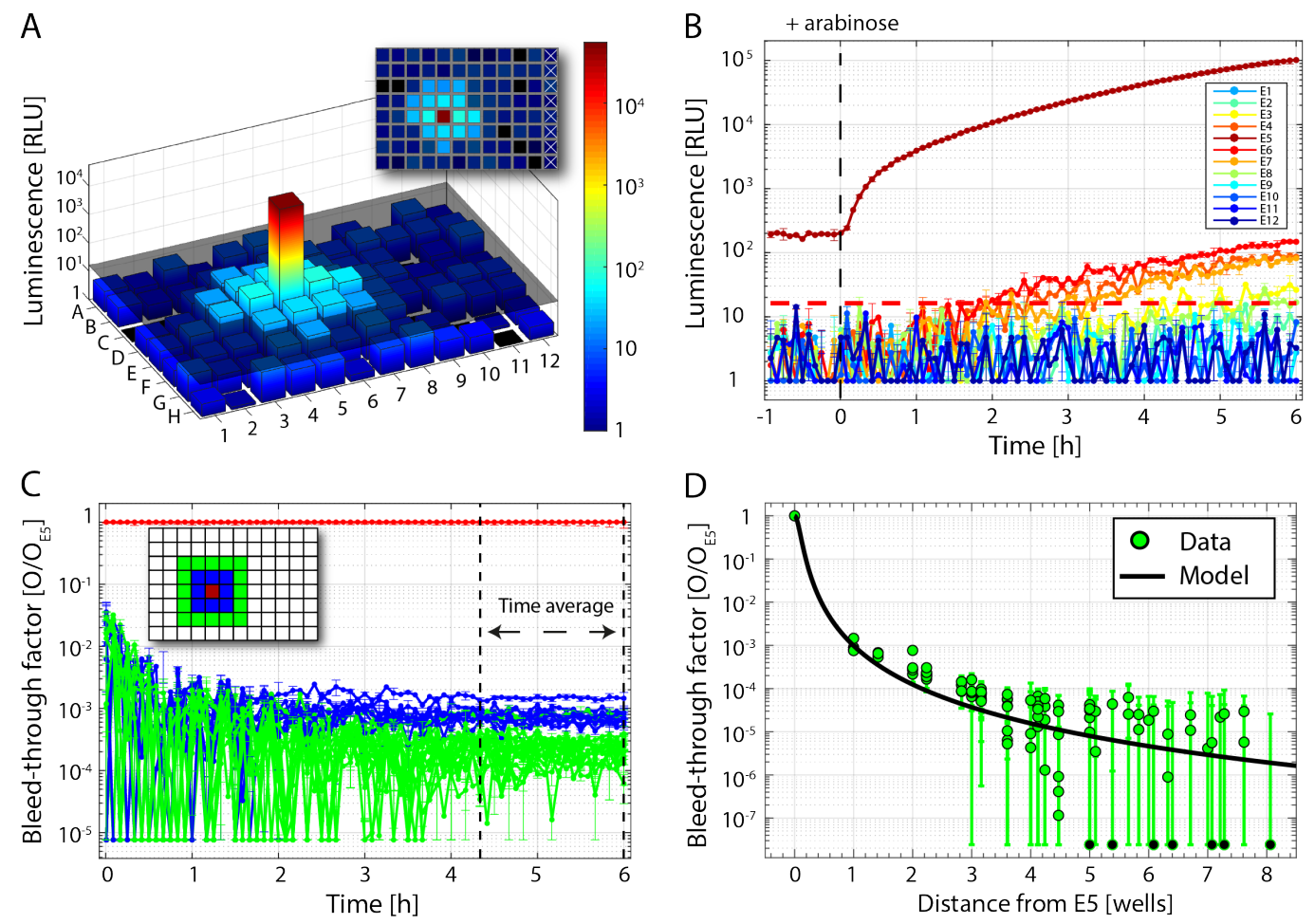

Figure S8: Quantification of luminescence bleed-through in the black calibration microplate for a calibration plate with low luminescence intensity. (A) The black calibration plate was prepared with a single mildly luminescent spot in the well E5. All data are background-subtracted and averaged over two experimental replicates. The background was obtained by averaging the signal of the wells indicated by the white crosses in the inset over the time before addition of arabinose (induction time). The 3D-plot shows the observed luminescence signal at 270 minutes after the induction with $2 \cdot 10^{-5} \%$ arabinose. (B) Time evolution of the observed luminescence signal of the E-row. The red line represents the instrument sensitivity value, defined as three times the standard deviation of the background value. (C) Luminescence bleed-through factor of the two shells closest to $E 5$, in blue and green. Values were obtained dividing the observed signal from a specific well by the signal recorded in $E 5$. The dashed lines indicate the time range over which the bleed-through was averaged to obtain in (D) the bleed-through factor as a function of the distance from E5 (green dots). The black solid line is the parameter free prediction of the bleed-through given by Equation 6 . 

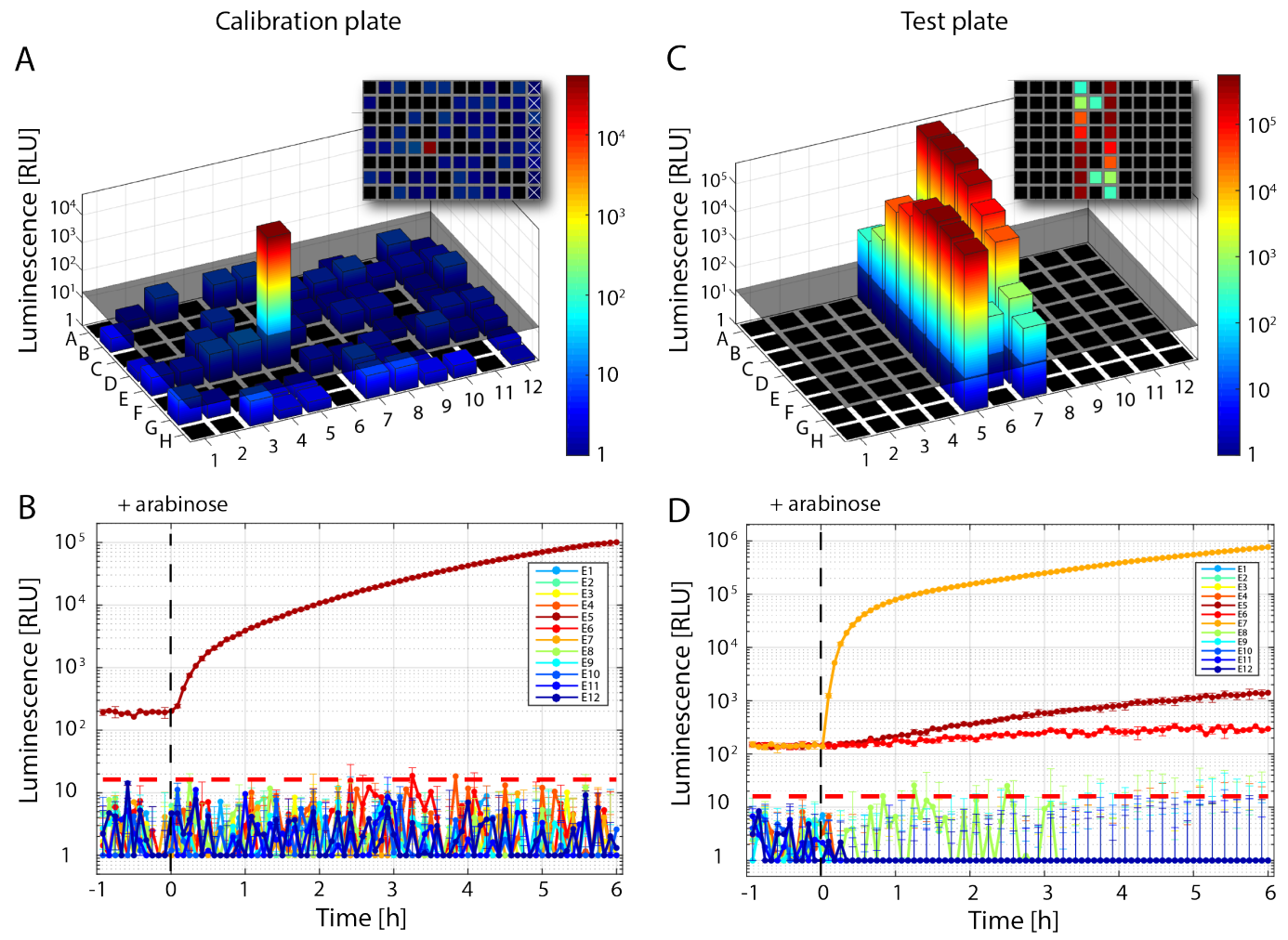

Figure S9: (A, B) Deconvolution of luminescence signals in the calibration microplate with low luminescence intensity. Luminescence values on the calibration plate 270 minutes after the induction of E. coli strain GFC0153 (in well E5) with with $2 \cdot 10^{-5 \%}$ (A) and its time evolution in the wells of row E after deconvolving by using the best kernel $D^{\text {best }}$ (B). (C, D) Deconvolution of luminescence signals in the test microplate by using the calibration plate with low luminescence intensity. Luminescence values on the test plate 270 minutes after the induction of $E$. coli strain GFC0153 with varying concentrations of arabinose (in wells A5-H5: $0 \%, 10^{-6 \%}, 10^{-5} \%, 2 \cdot 10^{-5} \%$, $5 \cdot 10^{-5} \%, 10^{-4 \%}, 10^{-3} \%, 2 \cdot 10^{-1} \%$; in wells A7-H7: $2 \cdot 10^{-1 \%}, 10^{-3 \%}, 10^{-4 \%}, 5 \cdot 10^{-5 \%}, 2 \cdot 10^{-5 \%}$, $10^{-5} \%, 10^{-6} \%, 0 \%$; in wells B6 and G6: $0 \%$ ) in C. The time evolution of luminescence values is shown in the wells of row $\mathrm{B}$ after deconvolving by using either the best kernel $D^{\text {best }}$ (in D). 

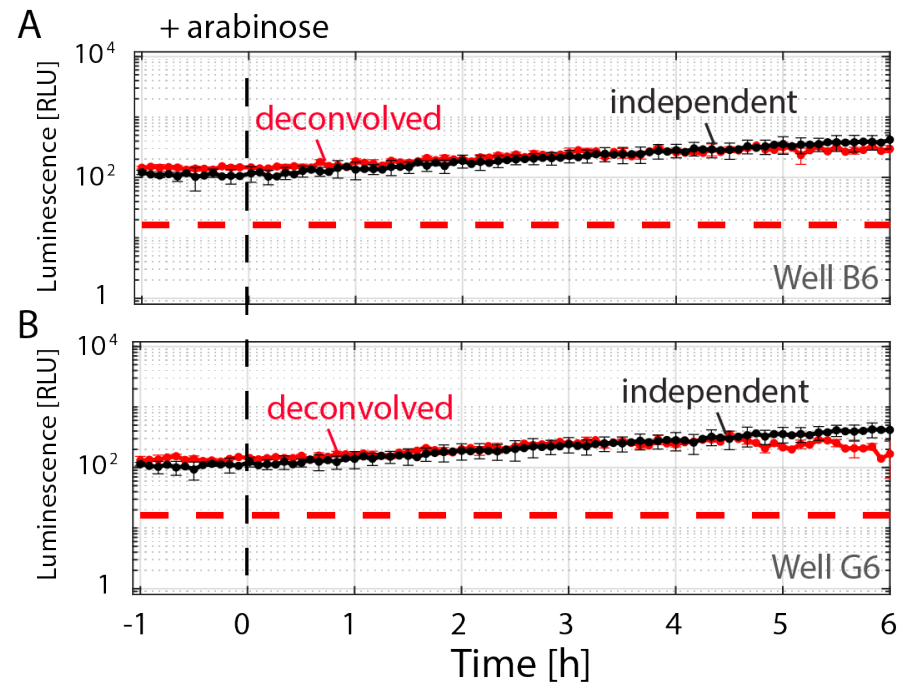

Figure S10: Comparison between deconvolution results and independent measurement. The luminescence values of wells B6 (panel A) and G6 (panel B) of the deconvolved test plate as compared to independent measurements in the absence of bleed-through, for which E. coli strain GFC0153 in wells B6 and G6 was not induced with arabinose, as described in the main text Figure 4. Black and red lines represent the independently measured and the deconvolved signal, respectively.

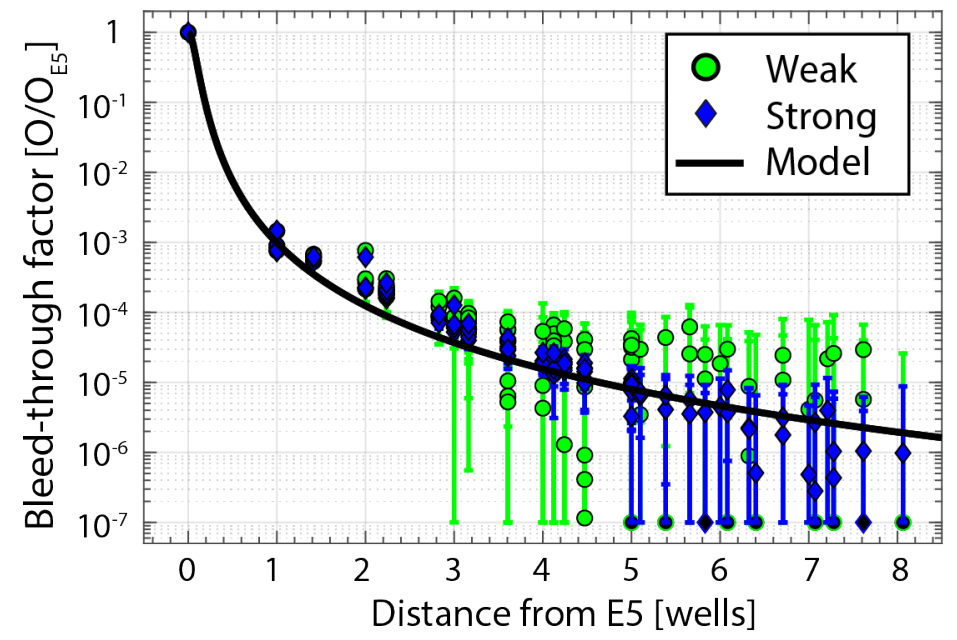

Figure S11: Comparison between bleed-through factor generated from the strongly induced luminescent strain (induced with $0.2 \%$ arabinose, from Figure 1 of the main text) and the weakly induced luminescent strain (induced with $2 \cdot 10^{-5 \%}$ arabinose, from Figure $\mathrm{S} 8$ ) as a function of the distance from $E 5$ as blue diamonds and green points, respectively. The figure shows that the bleed-through factor is independent of the luminescence signal strength, for values of the signal above the background. 

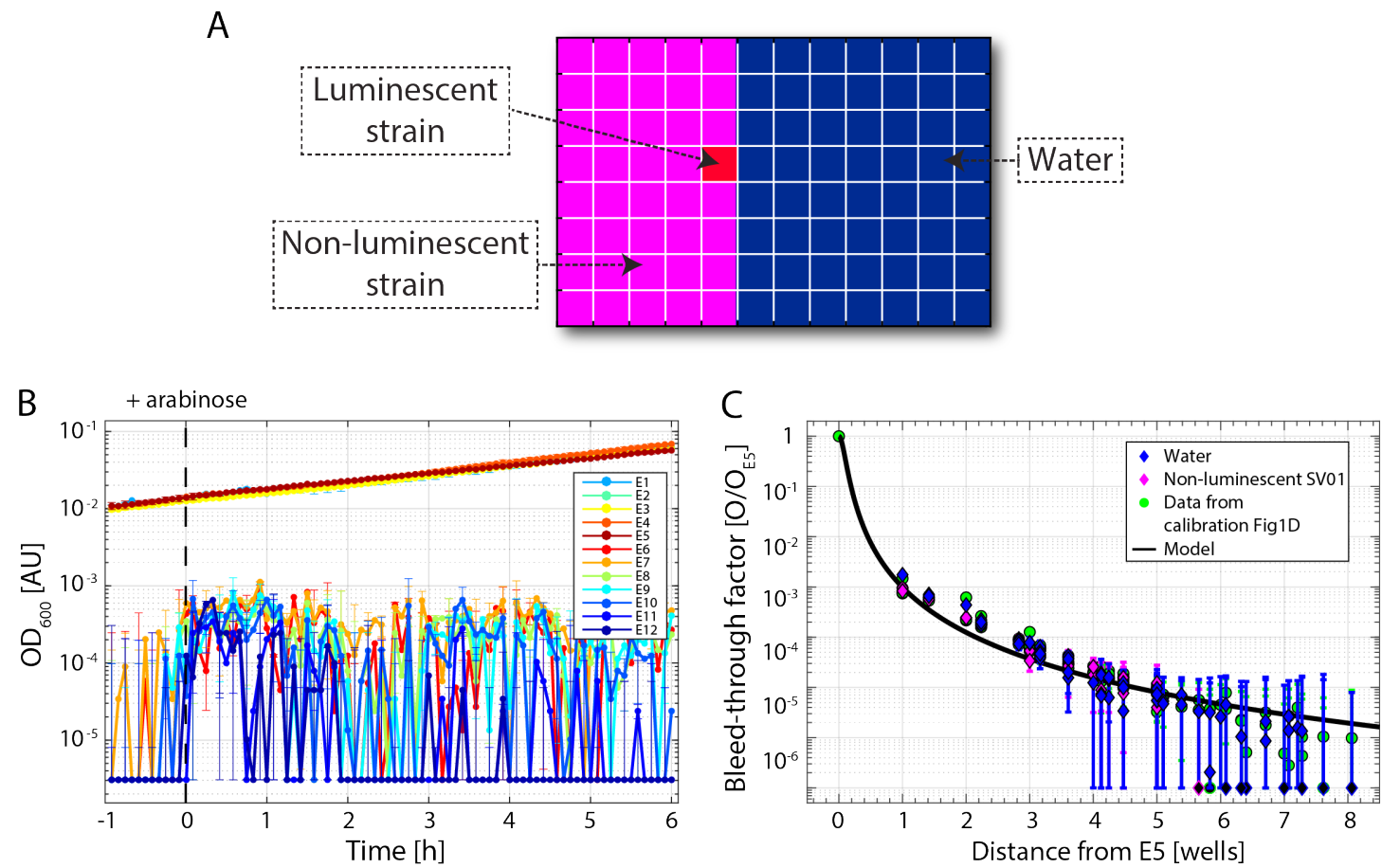

Figure S12: Panel A shows the arrangement of the calibration plate: left half (columns 1 to 5) was filled with the non-luminescent $E$. coli strain SV01 and right half (columns 6 to 12) with water. Water and bacterial strain SV01 have two order of magnitude difference in the measured OD $\left(10^{-4}\right.$ and $10^{-2}$, respectively), as shown from the OD measured along row $\mathrm{E}$ of the plate, in panel $\mathrm{B}$. Panel C demonstrates that bleed-through factors of water (blue diamonds) and SV01 (magenta diamonds) have same values of the calibration plate of Figure 1D in the main text (green points). 


\section{Control strain}

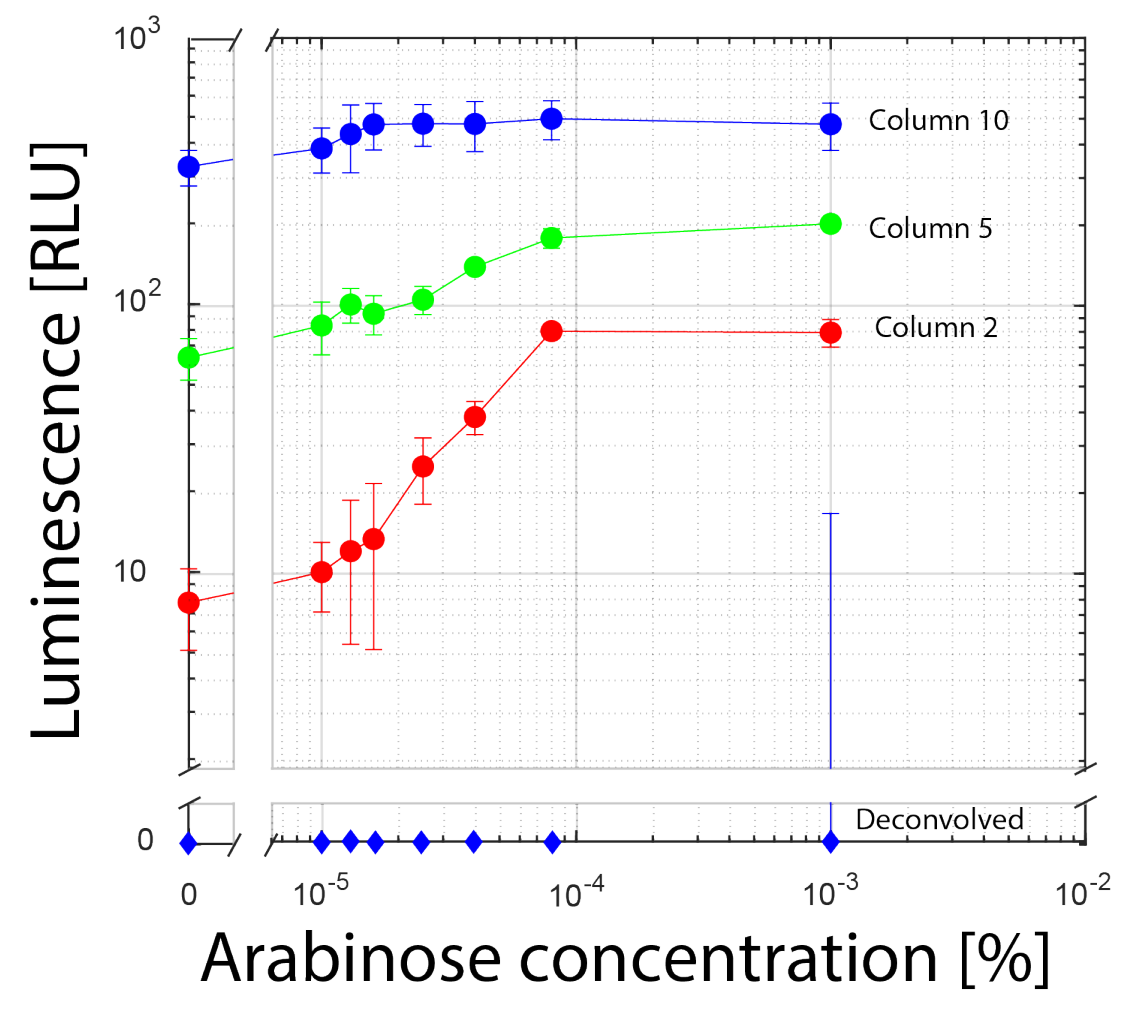

Figure S13: Dose-response of the bleed-through signals of the non-luminescent control strains in column 2, 5 and 10 of the plate in Figure 5 before (points) and after (diamonds) deconvolution process at 270 minutes after adding arabinose. 

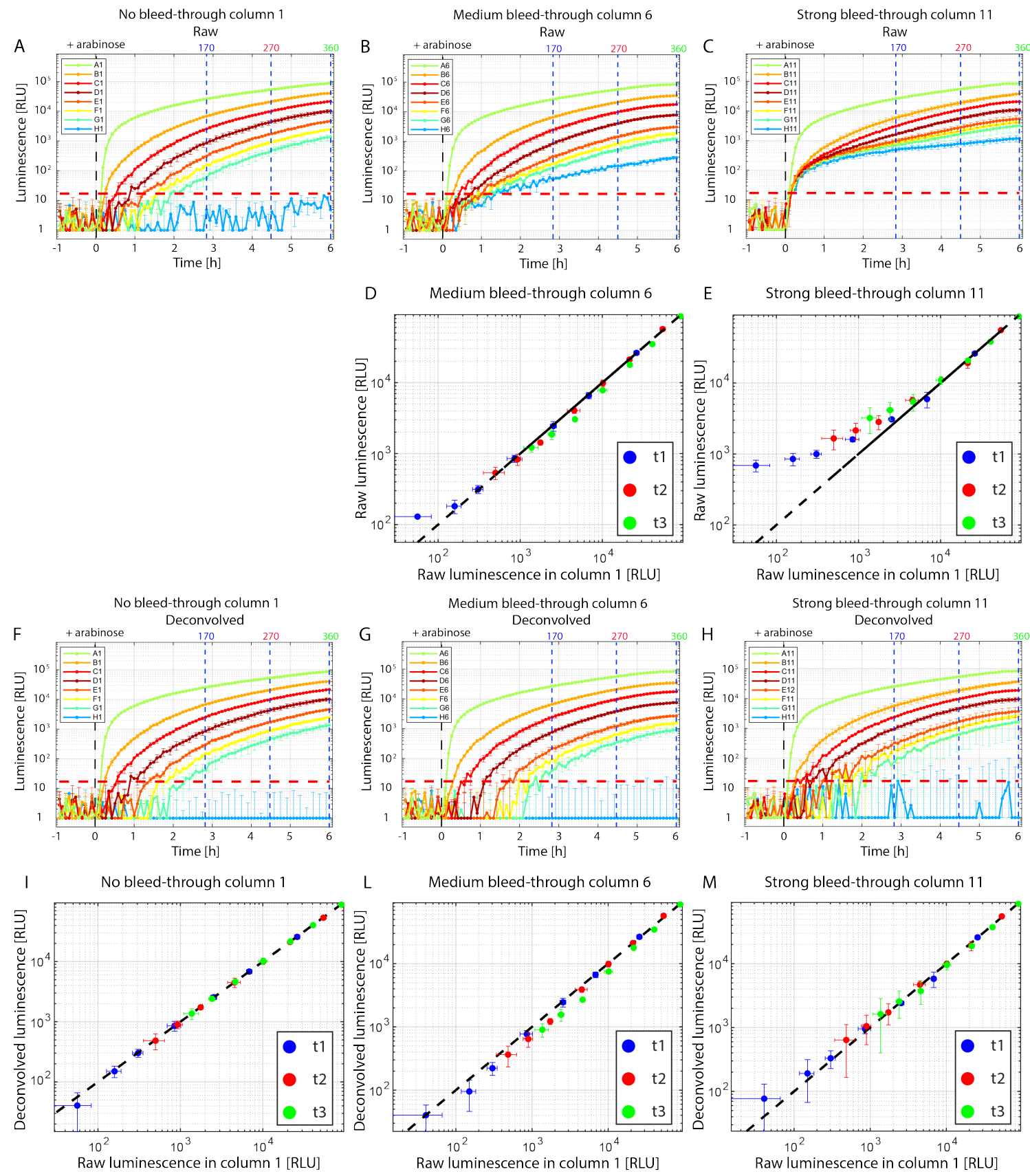

Figure S14: Time evolution of luminescence values of column 1 (no bleed-through, panel A raw and $\mathrm{F}$ deconvolved), of column 6 (medium bleed-through, panel B raw and G deconvolved) and of column 11 (strong bleed-through, panel $\mathrm{C}$ raw and $\mathrm{H}$ deconvolved) of Figure 5 in main text. Panels D, E are the scatter plot of raw luminescence of column 6 and 11 (medium and strong bleed-through, respectively) as function of raw luminescence of column 1. Panels I, L and M show the scatter plots of deconvolved luminescence of column 1 (no bleed-through, G), 6 (medium bleed-through, H) and 11 (strong bleed-through, I) as function of luminescence of column 1. Scatter plots are represented at three time points: 170 minutes in blue, 270 minutes in red and 360 minutes in green. The black dashed line has slope one. 

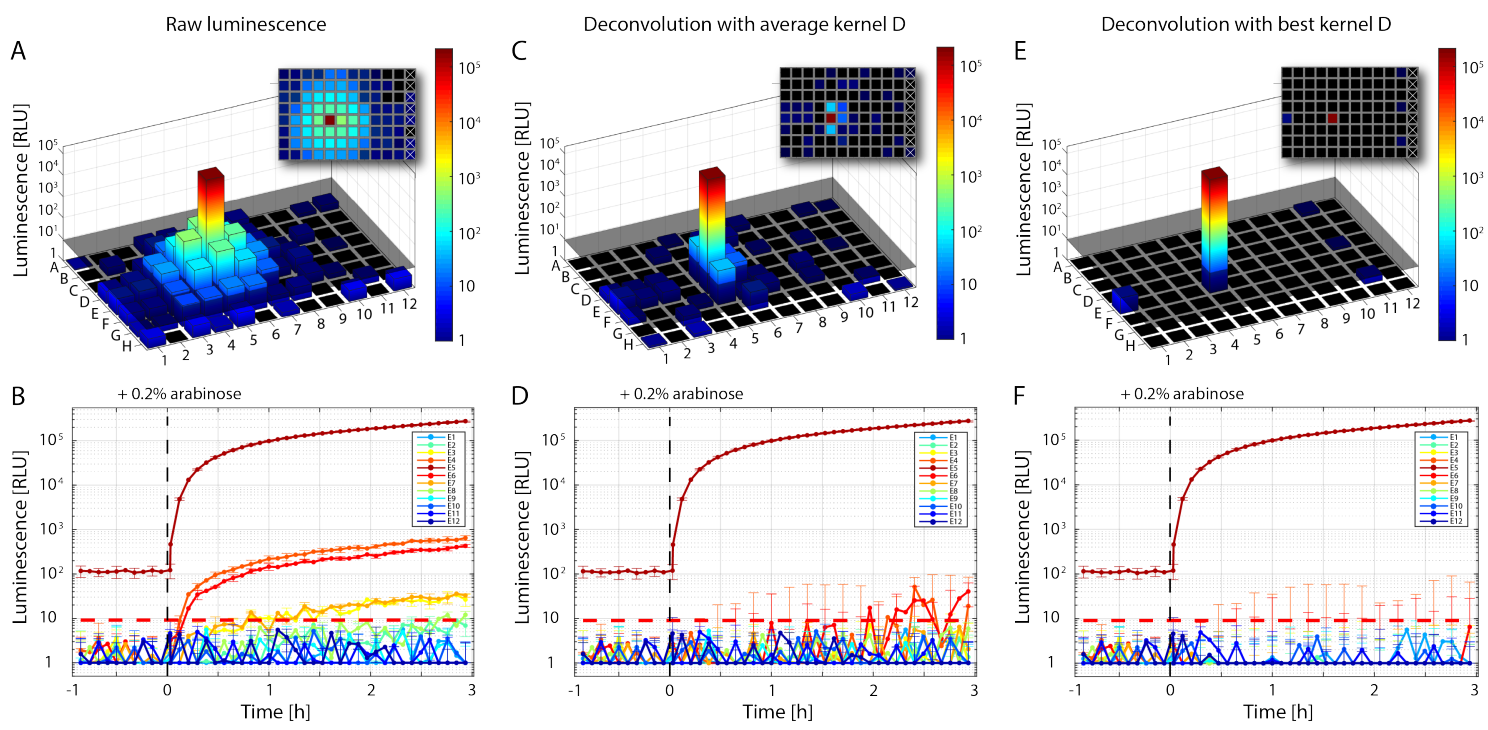

Figure S15: Calibration microplate in the VICTOR plate reader. Luminescence values on the calibration plate at 140 minutes after the induction (A, C and E) and its time evolution on the E-row (B, D and F) for raw signal (A, B), after deconvolving by using either the average kernel $D$ $(\mathrm{C}, \mathrm{D})$ or the best kernel $D_{\text {best }}(\mathrm{E}$ and F). 

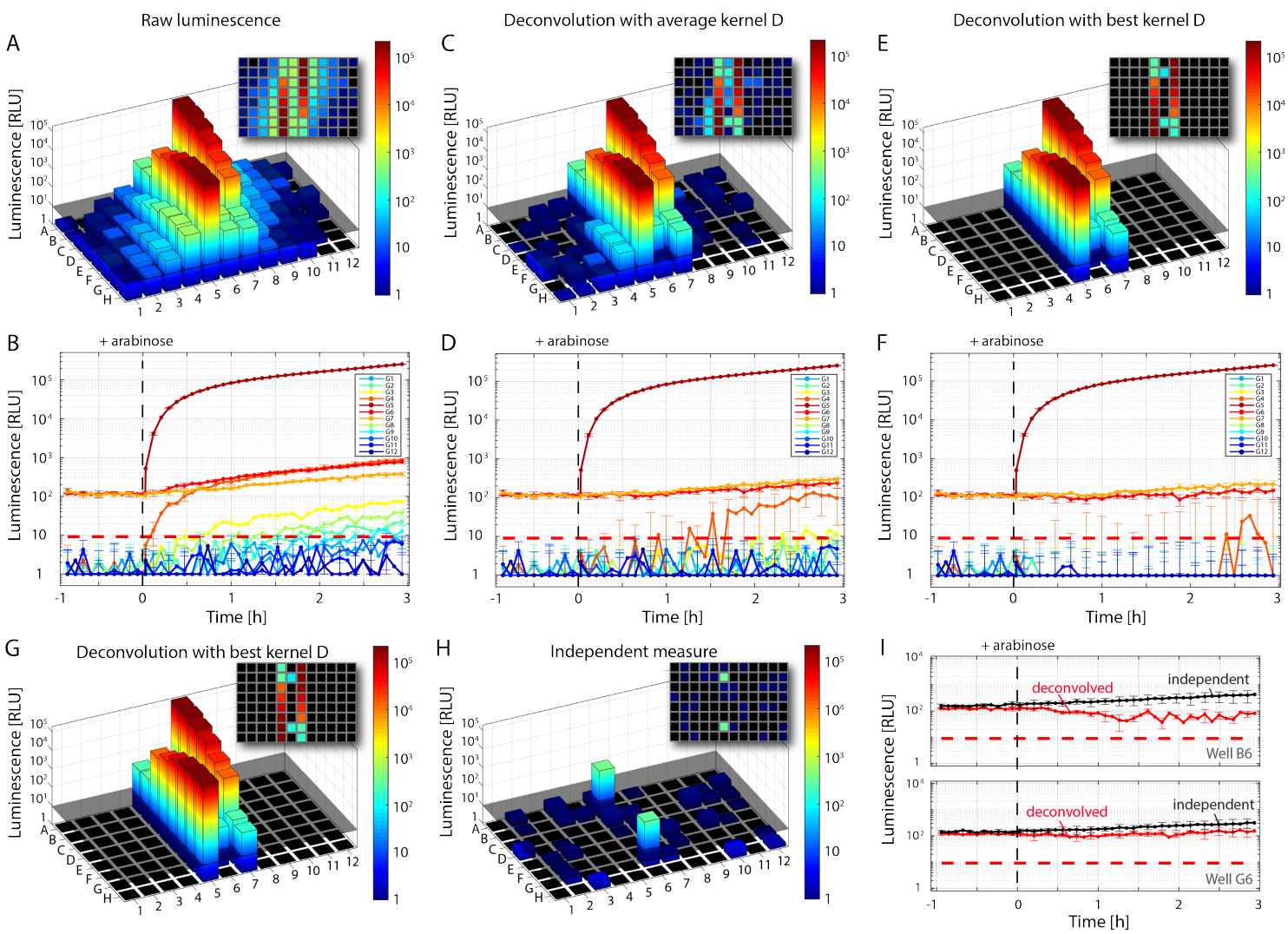

Figure S16: Test microplate in the VICTOR plate reader: luminescence values on the test plate at 270 minutes after the induction $(\mathrm{A}, \mathrm{C}$ and $\mathrm{E})$ and its time evolution on the G-row $(\mathrm{B}, \mathrm{D}$ and $\mathrm{F}$ ) for raw signal (A, B), after deconvolving by using either the average kernel $D(\mathrm{C}, \mathrm{D})$ or the best kernel $D_{\text {best }}(\mathrm{E}$ and F). Panels G H and I show the comparison between deconvolved test plate and independent measure. The luminescence values of wells B6 and G6 of the deconvolved test plate $(\mathrm{G})$ were compared to independent measure in the absence of bleed-through $(\mathrm{H})$. Black and red lines in (I) represent the independently measured and the deconvolved signal, respectively. 

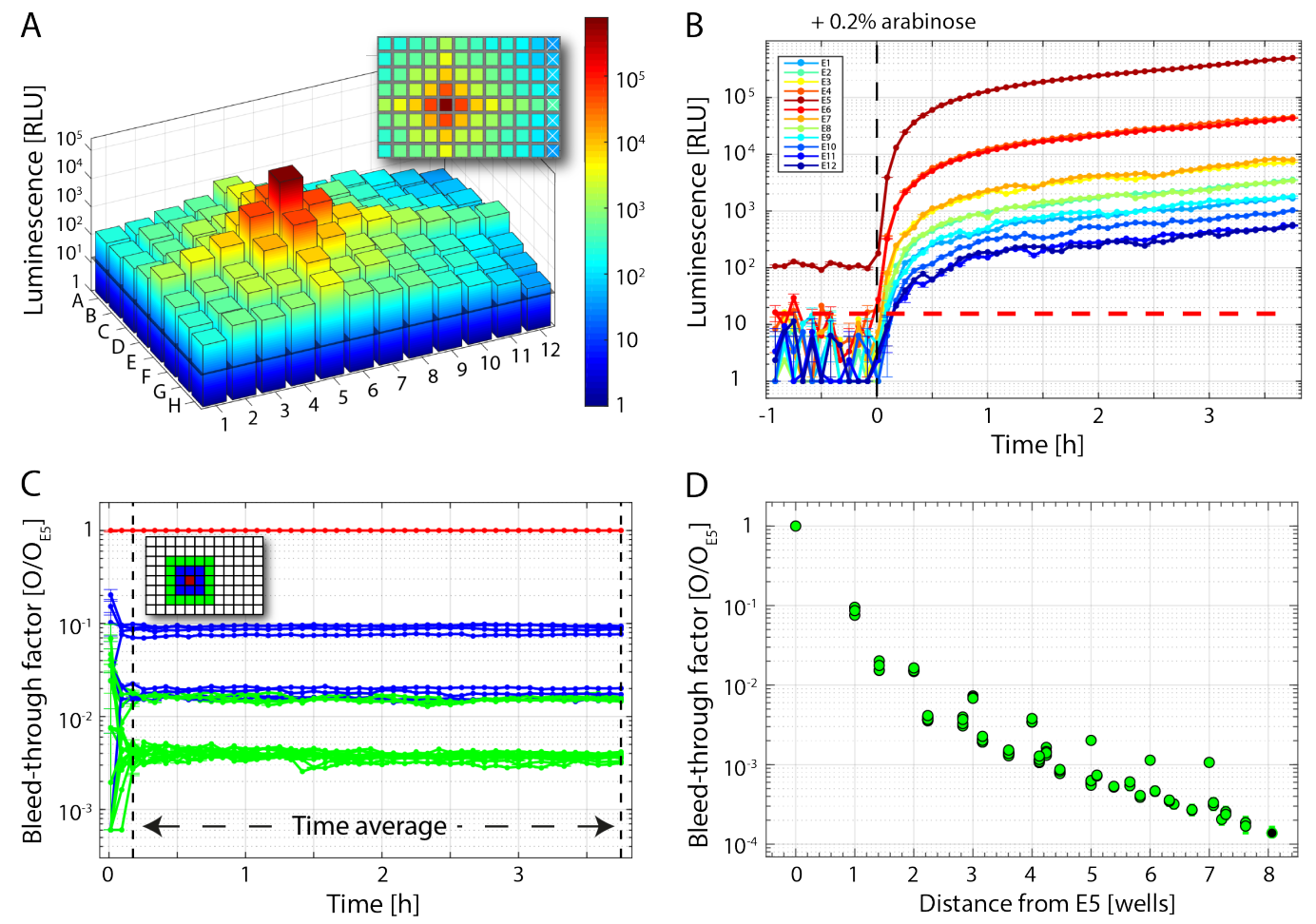

Figure S17: Quantification of luminescence bleed-through in the Tecan plate reader using a transparent microplate. (A) The calibration plate was prepared with a single highly luminescent spot in the well E5. All data are background-subtracted and averaged over three experimental replicates. The background was obtained by averaging the signal of the wells indicated by the white crosses in the inset over the time before addition of arabinose (induction time). The 3D-plot shows the observed luminescence signal at 135 minutes after the induction. (B) Time evolution of the observed luminescence signal of the E-row. The red line represents the instrument sensitivity value, defined as three times the standard deviation of the background value. (C) Luminescence bleed-through factor of the two shells closest to $E 5$, in blue and green. Values were obtained dividing the observed signal from a specific well by the signal recorded in E5. The dashed lines indicate the time range over which the bleed-through was averaged to obtain in (D) the bleed-through factor as a function of the distance from $E 5$ (green dots). 

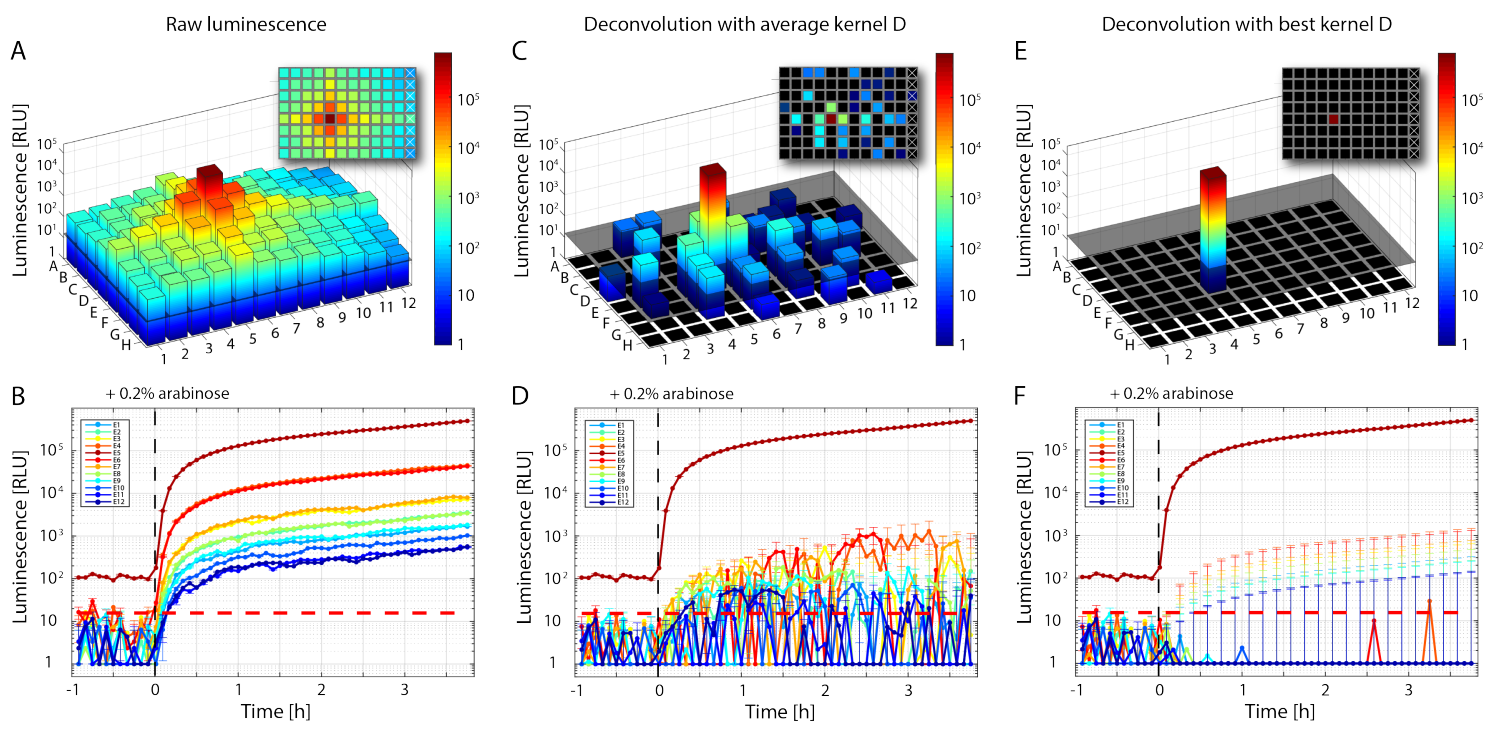

Figure S18: Calibration microplate in the Tecan plate reader with a transparent microplate. Luminescence values on the calibration plate at 135 minutes after the induction (A, C and $\mathrm{E})$ and its time evolution on the E-row (B, D and F) for raw signal (A, B), after deconvolving by using either the average kernel $D(\mathrm{C}, \mathrm{D})$ or the best kernel $D_{\text {best }}(\mathrm{E}$ and $\mathrm{F})$. 

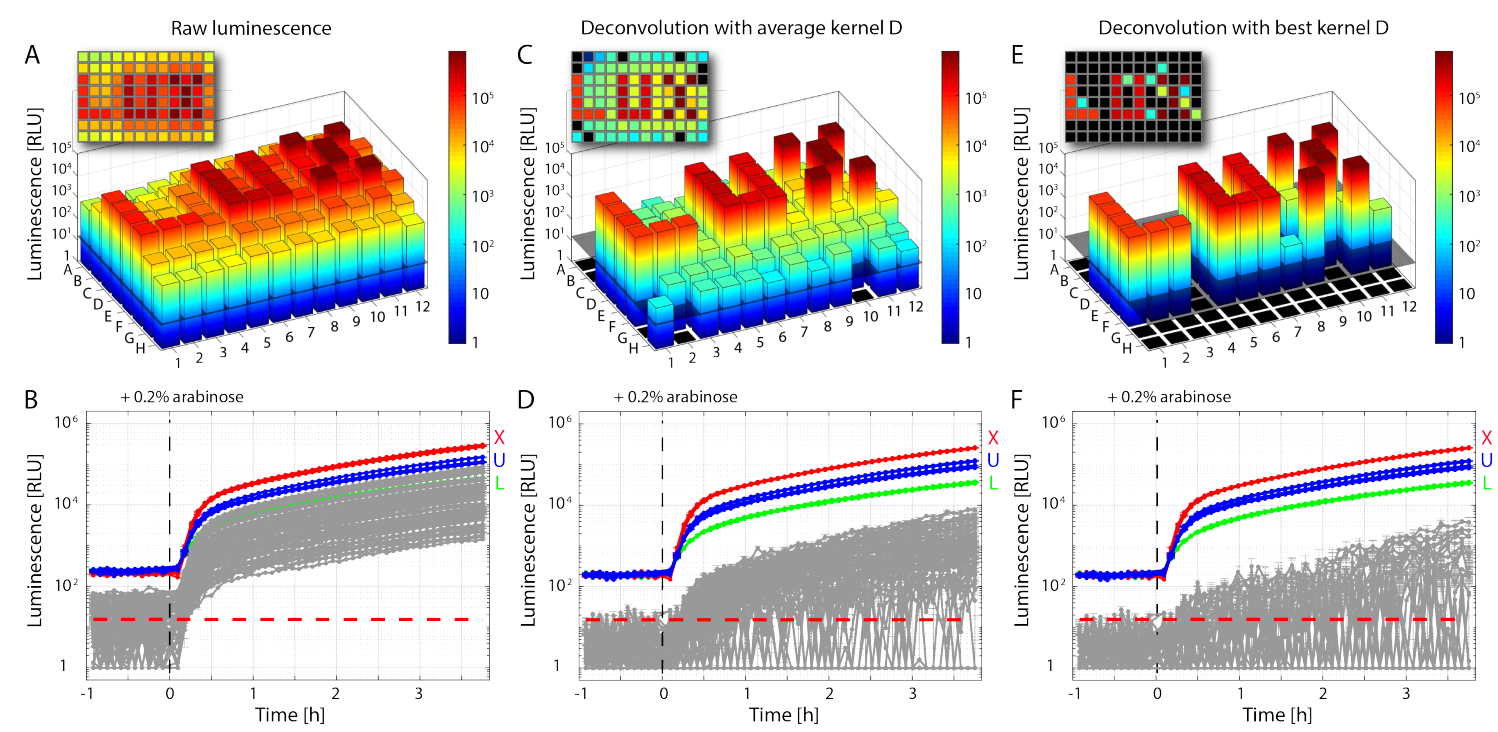

Figure S19: Test microplate in the Tecan plate reader with a transparent microplate. Luminescence values on the test plate at 135 minutes after the induction ( $\mathrm{A}, \mathrm{C}$ and $\mathrm{E}$ ) and its time evolution on the E-row (B, D and F) for raw signal (A, B), after deconvolving by using either the average kernel $D(\mathrm{C}, \mathrm{D})$ or the best kernel $D_{\text {best }}(\mathrm{E}$ and $\mathrm{F})$. In the test plate we arranged luminescent wells to compose the word LUX. In B, D and F the time evolution of the luminescence of L, U, X and remaining wells are represented in green, blue, red and gray, respectively. 


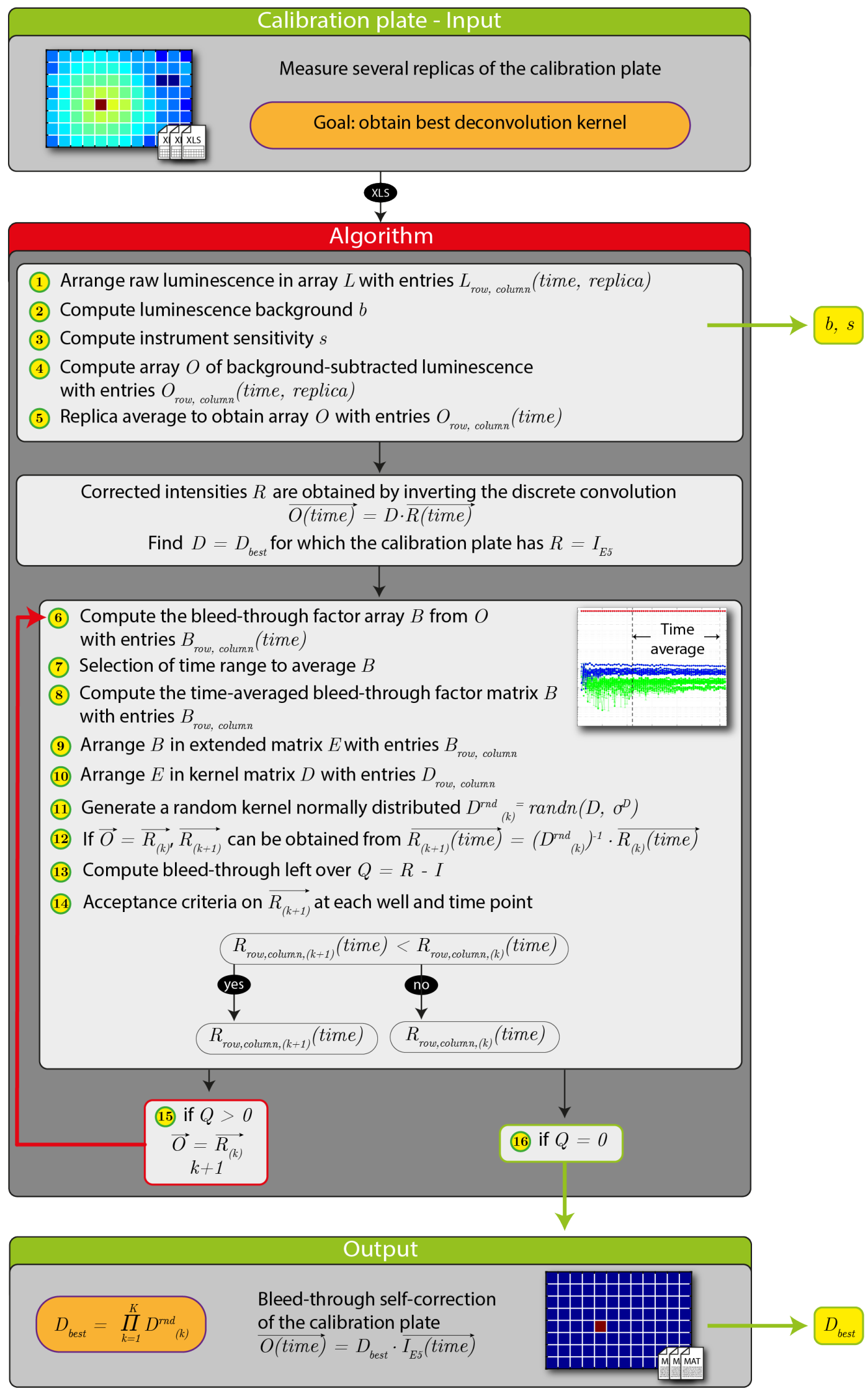

Figure S20: Workflow to find the best kernel $D_{\text {best }}$ from the calibration plate measurement. 


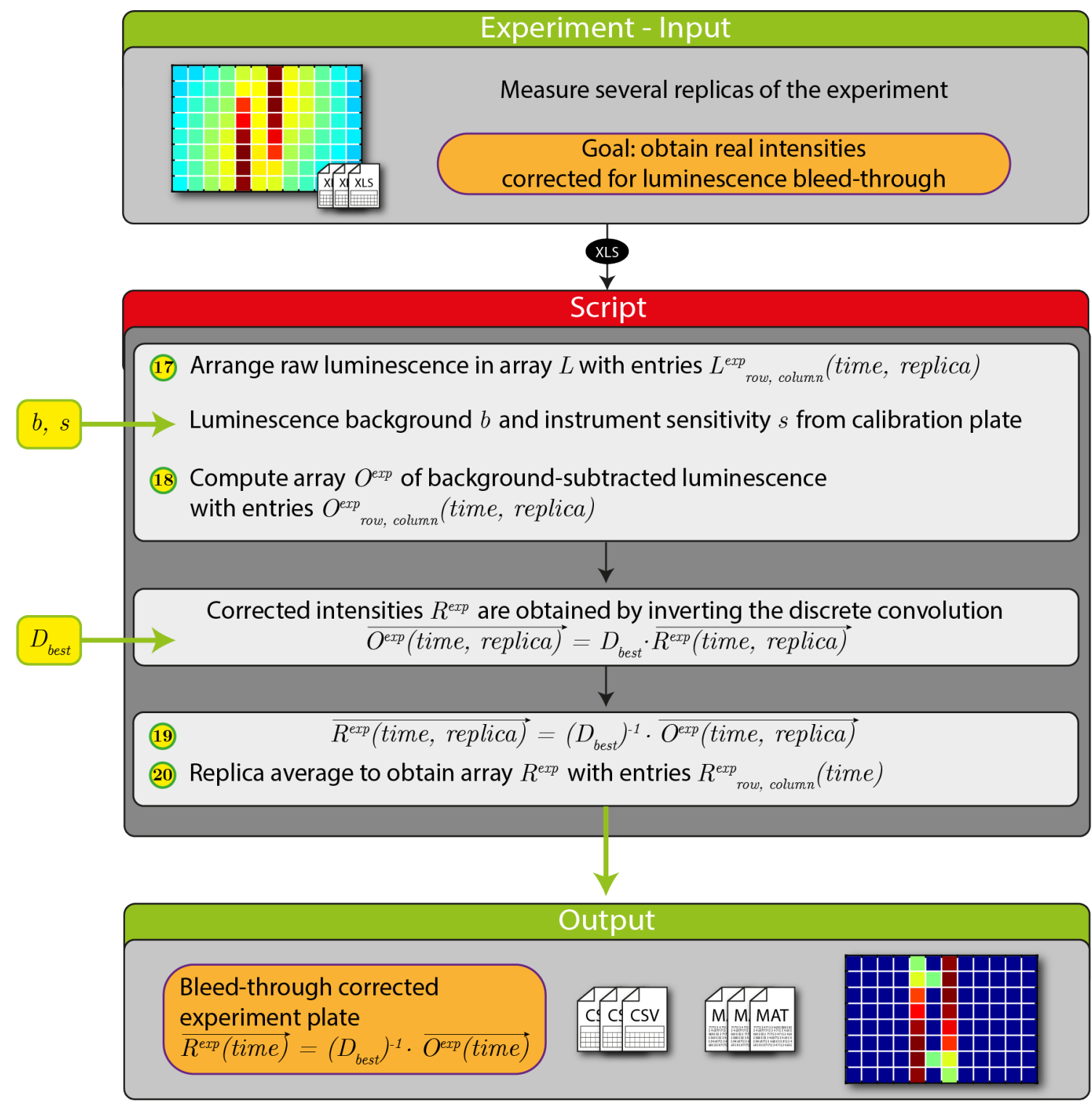

Figure S21: Workflow of the luminescence bleed-through correction of the experiment. 
Deconvolved calibration plate

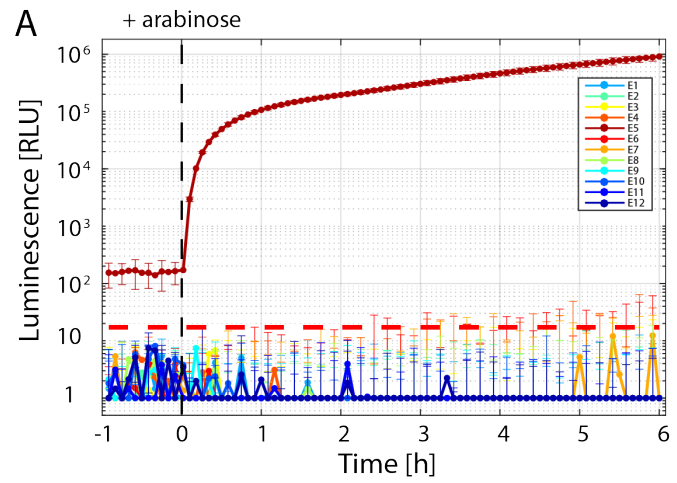

$\mathrm{OD}$
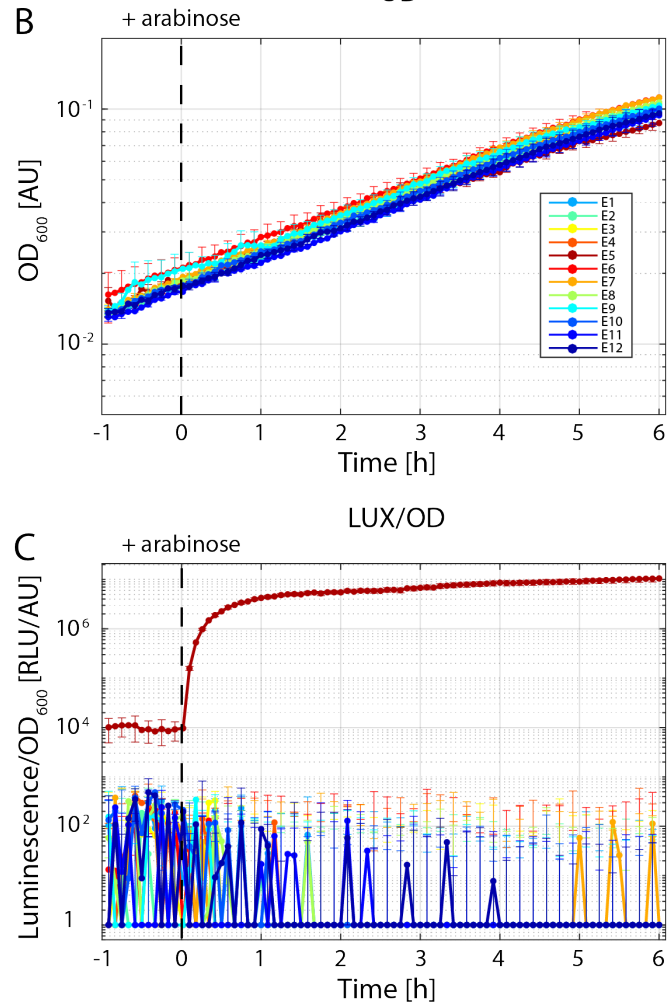

Deconvolved test plate

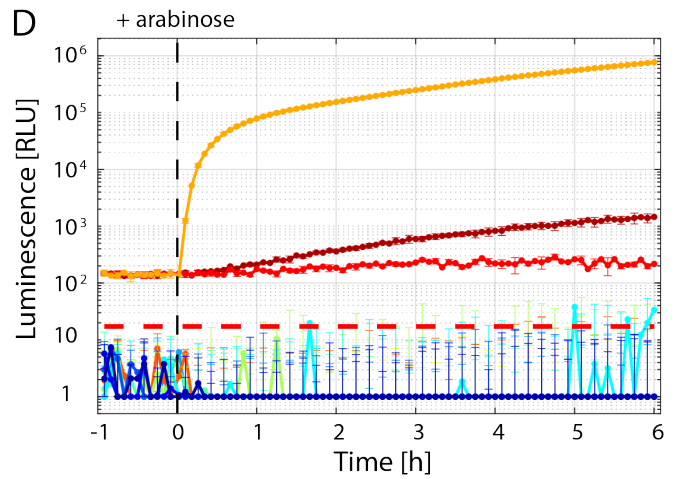

$\mathrm{OD}$
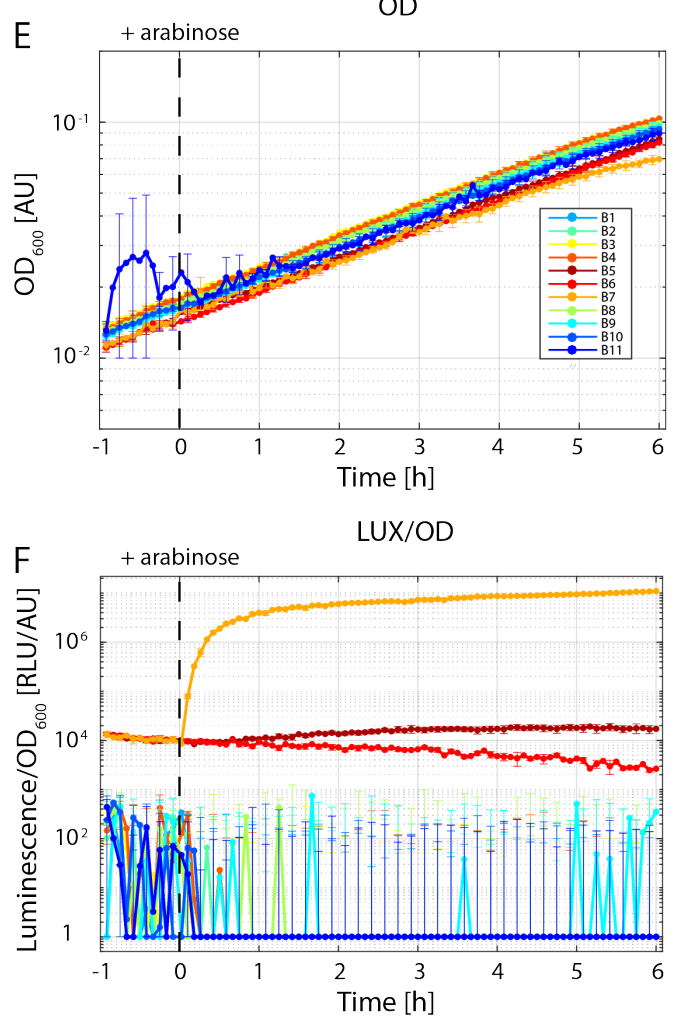

Figure S22: Deconvolved luminescence intensities (A and D), optical density (B and E) and deconvolved luminescence per optical density $(\mathrm{C}$ and $\mathrm{F}$ ) of the row $\mathrm{E}$ of the calibration plate of Figure 2 in the main text and of the row $B$ of the test plate of Figure 3 in the main text $(A, B, C$ and $\mathrm{D}, \mathrm{E}, \mathrm{F}$, respectively). 

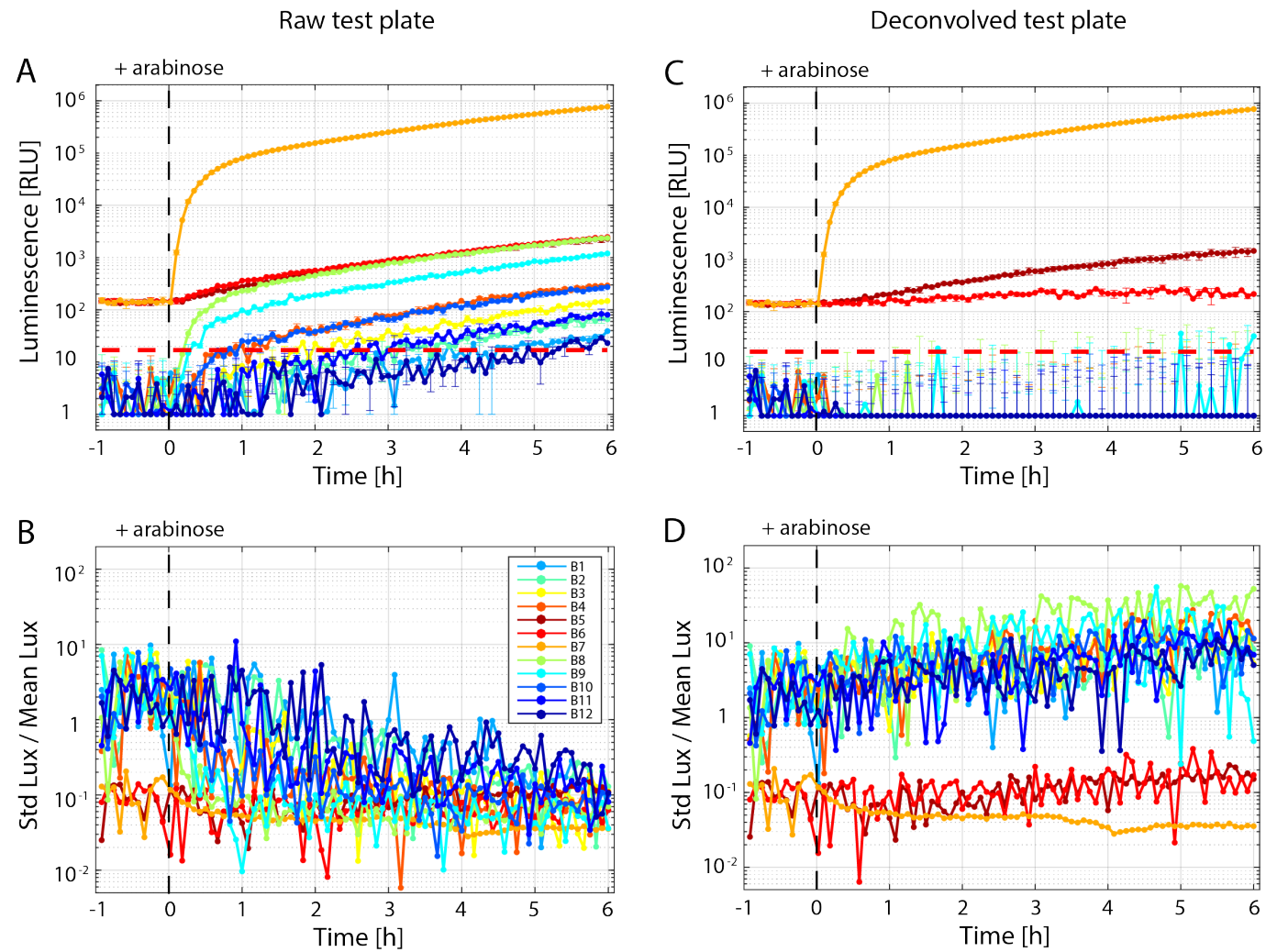

Figure S23: Raw (A) and deconvolved luminescence (C) of the row B of the test plate of Figure $3 \mathrm{~B}$ and $3 \mathrm{~F}$ in the main text. Panels $\mathrm{B}$ and $\mathrm{D}$ show the coefficient of variations (standard deviation over mean) corresponding to the data of $\mathrm{A}$ and $\mathrm{C}$, respectively. 


\section{A Calibration plate}

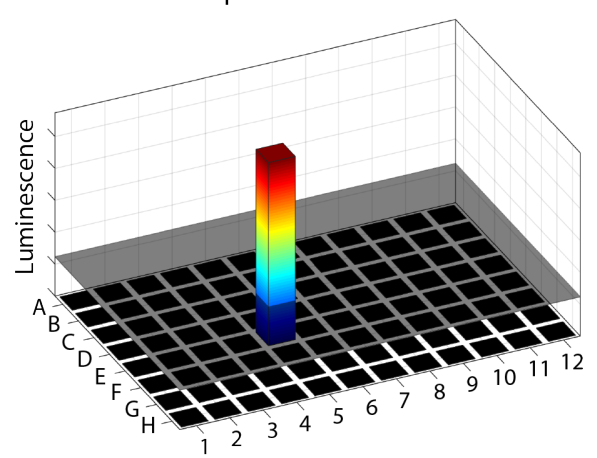

C Test plate

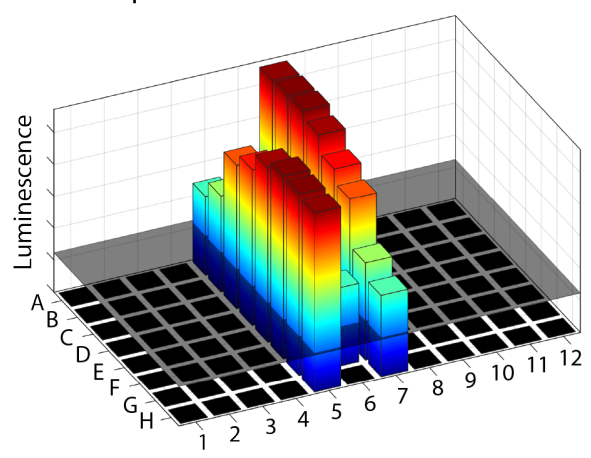

E Bleed-through strength plate

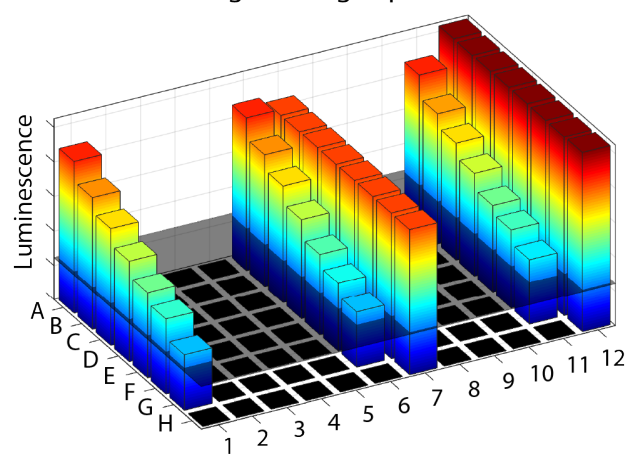

B

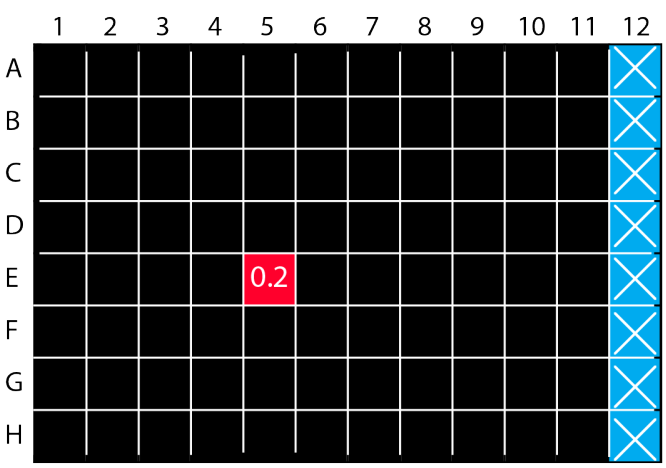

D

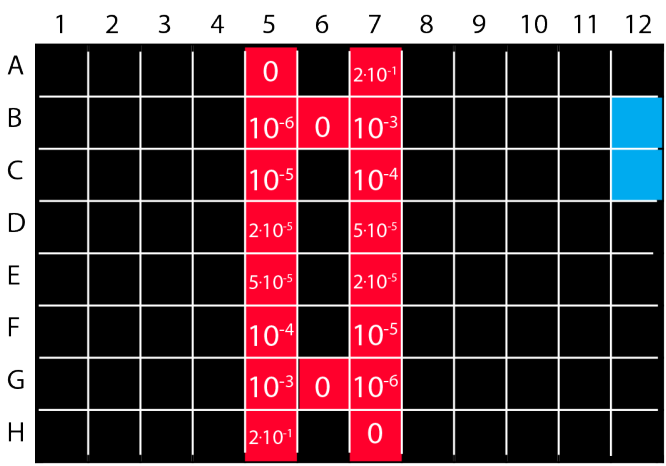

$\mathrm{F}$

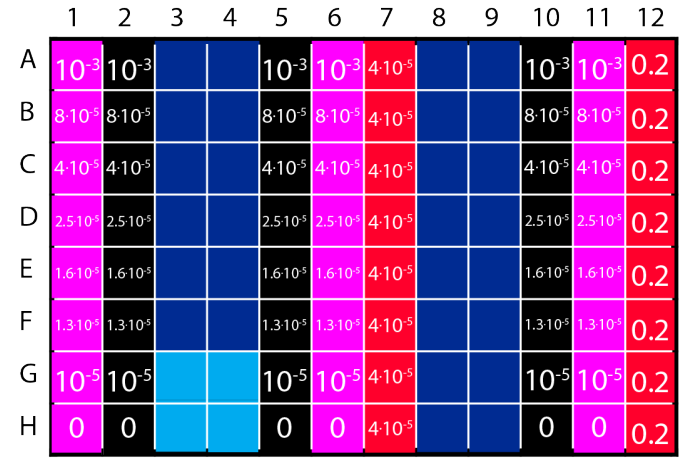

GFC0153 (LUX on plasmid) Luminescent strain
GFC208 (LUX on chromosome) Luminescent strain
SV01

Non luminescent strain

Medium:

Water

Well to obtain OD background

Well to obtain LUX background

Figure S24: Summary of the plate arrangements used in the experiments. 
A

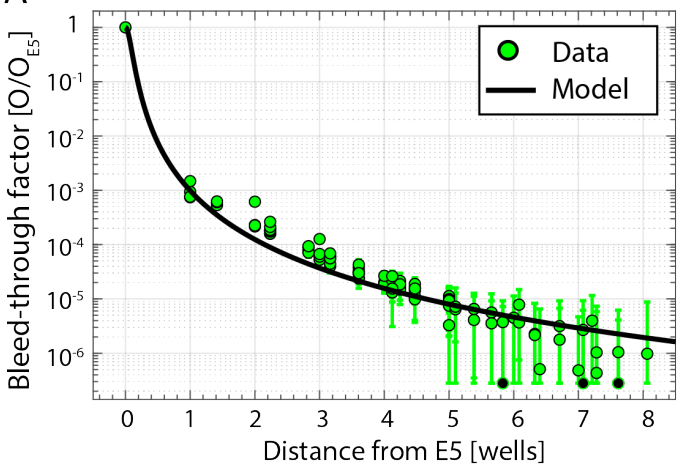

C

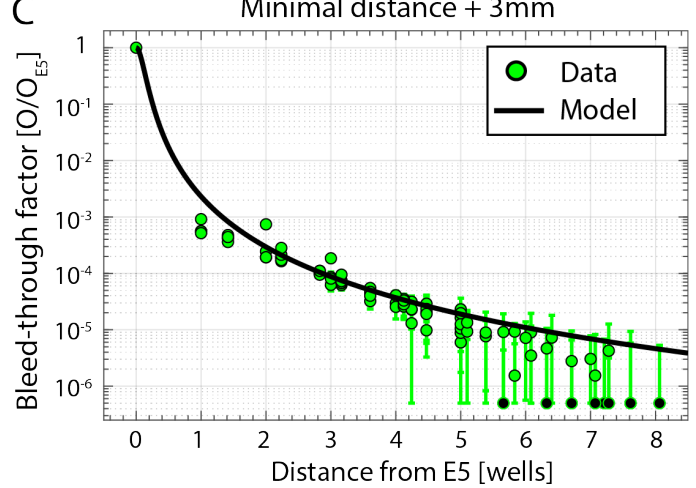

$\mathrm{E}$

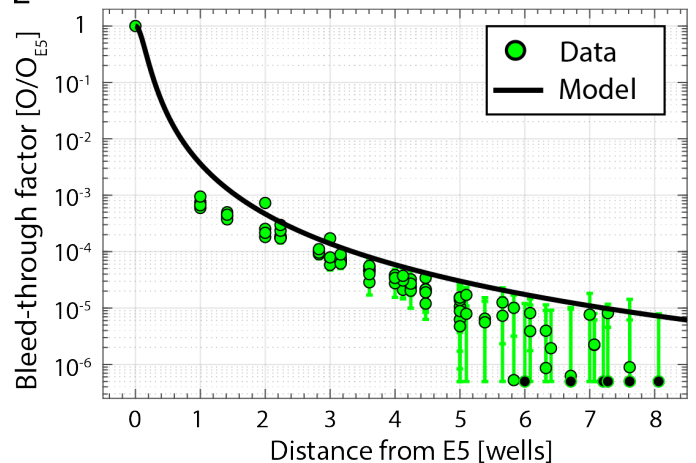

G

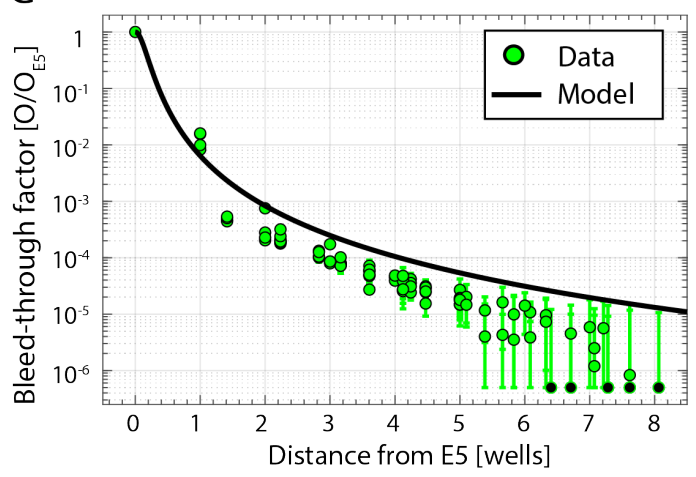

B
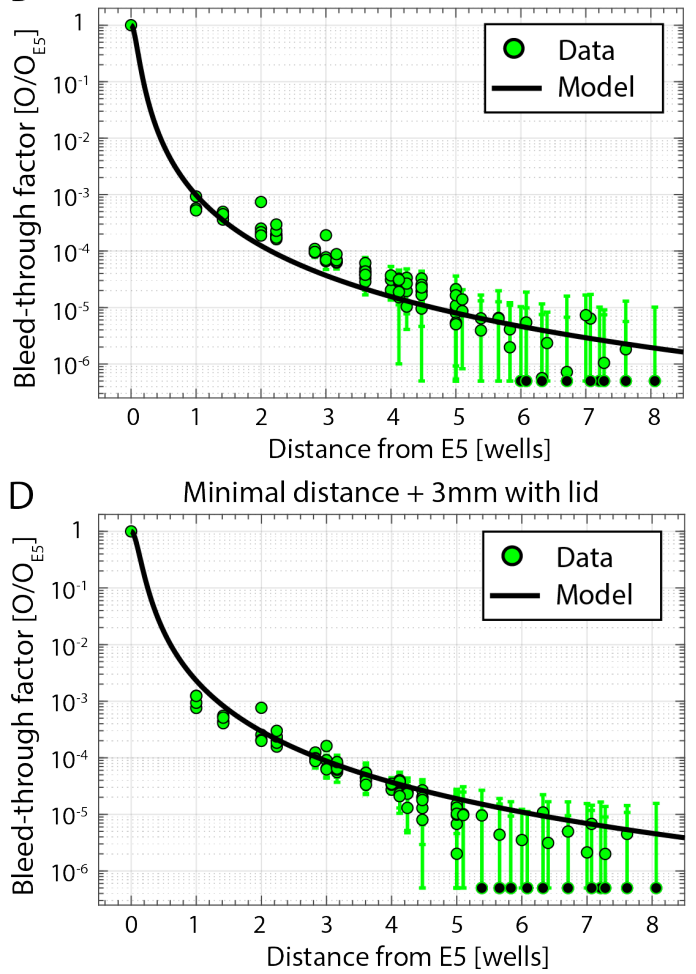

$\mathrm{F}$

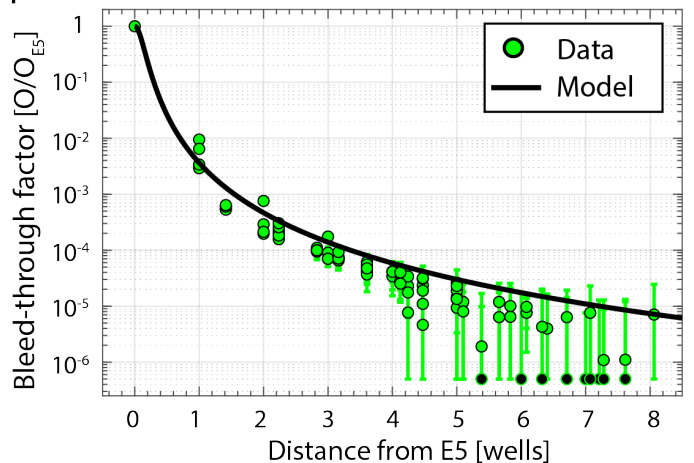

$\mathrm{H} \quad$ Comparison panel (A) and (G)

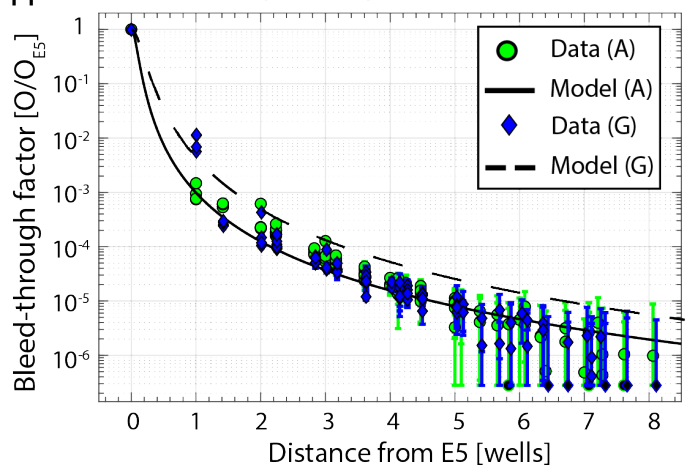

Figure S25: Bleed-through factor as a function of the distance from E5 in the case when the distance between the detector and the plate is minimal $(h=0.9 \mathrm{~mm}, \mathrm{~A})$, is minimal but the plate mounts a lid (B), is $3 \mathrm{~mm}$ above minimal distance without and with lid (C and $\mathrm{D}$, respectively), is 5 $\mathrm{mm}$ above minimal distance without and with lid ( $\mathrm{E}$ and $\mathrm{F}$, respectively), is $8 \mathrm{~mm}$ above minimal distance $(G)$. Data points and error bars represent mean and standard deviations from either two or three experimental replicates. The black solid line are the parameter free predictions of the bleedthrough given by Equation (2) in the main text drawn by modifying the parameter $h$ representing the distance between the plate and detector. $(\mathrm{H})$ Comparison between the minimal distance (green points) and $8 \mathrm{~mm}$ above the minimal distance (blue diamonds), as represented in $(\mathrm{A})$ and in $(\mathrm{G})$, respectively. 


\section{DNA sequence of pSVM-MC_038}

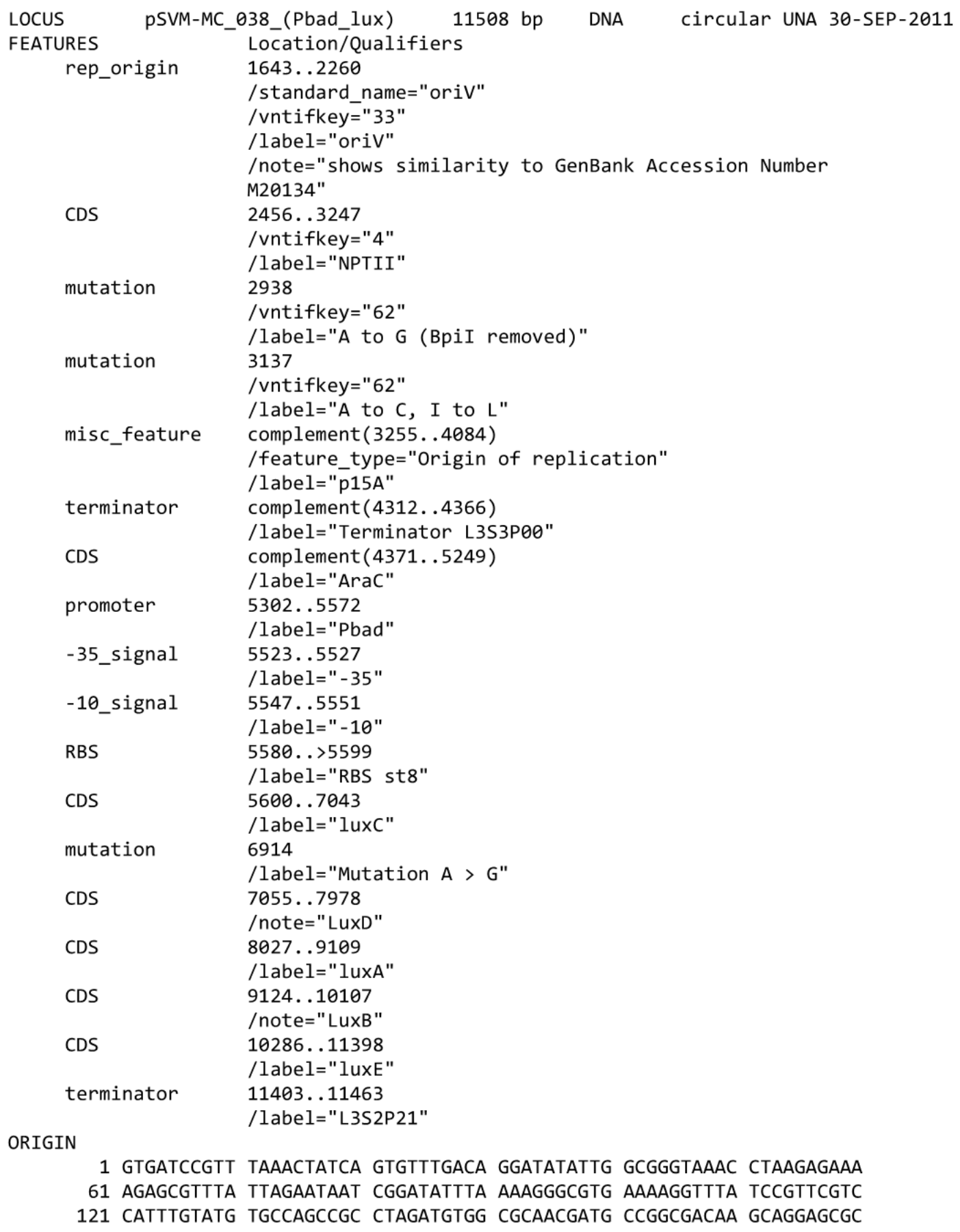

1 GTGATCCGTT TAAACTATCA GTGTTTGACA GGATATATTG GCGGGTAAAC CTAAGAGAAA 61 AGAGCGTTTA TTAGAATAAT CGGATATTTA AAAGGGCGTG AAAAGGTTTA TCCGTTCGTC 121 CATTTGTATG TGCCAGCCGC CTAGATGTGG CGCAACGATG CCGGCGACAA GCAGGAGCGC 
181 ACCGACTTCT TCCGCATCAA GTGTTTTGGC TCTCAGGCCG AGGCCCACGG CAAGTATTTG 241 GGCAAGGGGT CGCTGGTATT CGTGCAGGGC AAGATTCGGA ATACCAAGTA CGAGAAGGAC 301 GGCCAGACGG TCTACGGGAC CGACTTCATT GCCGATAAGG TGGATTATCT GGACACCAAG 361 GCACCAGGCG GGTCAAATCA GGAATAAGGG CACATTGCCC CGGCGTGAGT CGGGGCAATC 421 CCGCAAGGAG GGTGAATGAA TCGGACGTTT GACCGGAAGG CATACAGGCA AGAACTGATC 481 GACGCGGGGT TTTCCGCCGA GGATGCCGAA ACCATCGCAA GCCGCACCGT CATGCGTGCG 541 CCCCGCGAAA CCTTCCAGTC CGTCGGCTCG ATGGTCCAGC AAGCTACGGC CAAGATCGAG 601 CGCGACAGCG TGCAACTGGC TCCCCCTGCC CTGCCCGCGC CATCGGCCGC CGTGGAGCGT 661 TCGCGTCGTC TTGAACAGGA GGCGGCAGGT TTGGCGAAGT CGATGACCAT CGACACGCGA 721 GGAACTATGA CGACCAAGAA GCGAAAAACC GCCGGCGAGG ACCTGGCAAA ACAGGTCAGC 781 GAGGCCAAGC AGGCCGCGTT GCTGAAACAC ACGAAGCAGC AGATCAAGGA AATGCAGCTT 841 TCCTTGTTCG ATATTGCGCC GTGGCCGGAC ACGATGCGAG CGATGCCAAA CGACACGGCC 901 CGCTCTGCCC TGTTCACCAC GCGCAACAAG AAAATCCCGC GCGAGGCGCT GCAAAACAAG 961 GTCATTTTCC ACGTCAACAA GGACGTGAAG ATCACCTACA CCGGCGTCGA GCTGCGGGCC 1021 GACGATGACG AACTGGTGTG GCAGCAGGTG TTGGAGTACG CGAAGCGCAC CCCTATCGGC 1081 GAGCCGATCA CCTTCACGTT CTACGAGCTT TGCCAGGACC TGGGCTGGTC GATCAATGGC 1141 CGGTATTACA CGAAGGCCGA GGAATGCCTG TCGCGCCTAC AGGCGACGGC GATGGGCTTC 1201 ACGTCCGACC GCGTTGGGCA CCTGGAATCG GTGTCGCTGC TGCACCGCTT CCGCGTCCTG 1261 GACCGTGGCA AGAAAACGTC CCGTTGCCAG GTCCTGATCG ACGAGGAAAT CGTCGTGCTG 1321 TTTGCTGGCG ACCACTACAC GAAATTCATA TGGGAGAAGT ACCGCAAGCT GTCGCCGACG 1381 GCCCGACGGA TGTTCGACTA TTTCAGCTCG CACCGGGAGC CGTACCCGCT CAAGCTGGAA 1441 ACCTTCCGCC TCATGTGCGG ATCGGATTCC ACCCGCGTGA AGAAGTGGCG CGAGCAGGTC 1501 GGCGAAGCCT GCGAAGAGTT GCGAGGCAGC GGCCTGGTGG AACACGCCTG GGTCAATGAT 1561 GACCTGGTGC ATTGCAAACG CTAGGGCCTT GTGGGGTCAG TTCCGGCTGG GGGTTCAGCA 1621 GCCCAACCCT TTGCGACGCT CACCGGGCTG GTTGCCCTCG CCGCTGGGCT GGCGGCCGTC 1681 TATGGCCCTG CAAACGCGCC AGAAACGCCG TCGAAGCCGT GTGCGAGACA CCGCGGCCGC 1741 CGGCGTTGTG GATACCTCGC GGAAAACTTG GCCCTCACTG ACAGATGAGG GGCGGACGTT 1801 GACACTTGAG GGGCCGACTC ACCCGGCGCG GCGTTGACAG ATGAGGGGCA GGCTCGATTT 1861 CGGCCGGCGA CGTGGAGCTG GCCAGCCTCG CAAATCGGCG AAAACGCCTG ATTTTACGCG 1921 AGTTTCCCAC AGATGATGTG GACAAGCCTG GGGATAAGTG CCCTGCGGTA TTGACACTTG 1981 AGGGGCGCGA CTACTGACAG ATGAGGGGCG CGATCCTTGA CACTTGAGGG GCAGAGTGCT 2041 GACAGATGAG GGGCGCACCT ATTGACATTT GAGGGGCTGT CCACAGGCAG AAAATCCAGC 2101 ATTTGCAAGG GTTTCCGCCC GTTTTTCGGC CACCGCTAAC CTGTCTTTTA ACCTGCTTTT 2161 AAACCAATAT TTATAAACCT TGTTTTTAAC CAGGGCTGCG CCCTGTGCGC GTGACCGCGC 2221 ACGCCGAAGG GGGGTGCCCC CCCTTCTCGA ACCCTCCCGG CCCGCTAACG CGGGCCTCCC 2281 ATCCCCCCAG GGGCTGCGCC CCTCGGCCGC GAACGGCCTC ACCCCAAAAA TGGCAGCGCT 2341 GGCCCGAGTG CGCGGAACCC CTATTTGTTT ATTTTTCTAA ATACATTCAA ATATGTATCC 2401 GCTCATGAGA CAATAACCCT GATAAATGCT TCAATAATAT TGAAAAAGGA AGAGTATGGC 2461 TAAAATGAGA ATATCACCGG AATTGAAAAA ACTGATCGAA AAATACCGCT GCGTAAAAGA 2521 TACGGAAGGA ATGTCTCCTG CTAAGGTATA TAAGCTGGTG GGAGAAAATG AAAACCTATA 2581 TTTAAAAATG ACGGACAGCC GGTATAAAGG GACCACCTAT GATGTGGAAC GGGAAAAGGA 2641 CATGATGCTA TGGCTGGAAG GAAAGCTGCC TGTTCCAAAG GTCCTGCACT TTGAACGGCA 2701 TGATGGCTGG AGCAATCTGC TCATGAGTGA GGCCGATGGC GTCCTTTGCT CGGAAGAGTA 2761 TGAAGATGAA CAAAGCCCTG AAAAGATTAT CGAGCTGTAT GCGGAGTGCA TCAGGCTCTT 2821 TCACTCCATC GACATATCGG ATTGTCCCTA TACGAATAGC TTAGACAGCC GCTTAGCCGA 2881 ATTGGATTAC TTACTGAATA ACGATCTGGC CGATGTGGAT TGCGAAAACT GGGAAGAGGA 2941 CACTCCATTT AAAGATCCGC GCGAGCTGTA TGATTTTTTAA AAGACGGAAA AGCCCGAAGA 3001 GGAACTTGTC TTTTCCCACG GCGACCTGGG AGACAGCAAC ATCTTTGTGA AAGATGGCAA 3061 AGTAAGTGGC TTTATTGATC TTGGGAGAAG CGGCAGGGCG GACAAGTGGT ATGACATTGC 3121 CTTCTGCGTC CGGTCGCTCA GGGAGGATAT CGGGGAAGAA CAGTATGTCG AGCTATTTTT 
3181 TGACTTACTG GGGATCAAGC CTGATTGGGA GAAAATAAAA TATTATATTT TACTGGATGA 3241 ATTGTTTTAG CTGTTTAATA AGATGATCTT CTTGAGATCG TTTTGGTCTG CGCGTAATCT 3301 CTTGCTCTGA AAACGAAAAA ACCGCCTTGC AGGGCGGTTT TTCGAAGGTT CTCTGAGCTA 3361 CCAACTCTTT GAACCGAGGT AACTGGCTTG GAGGAGCGCA GTCACCAAAA CTTGTCCTTT 3421 CAGTTTAGCC TTAACCGGCG CATGACTTCA AGACTAACTC CTCTAAATCA ATTACCAGTG 3481 GCTGCTGCCA GTGGTGCTTT TGCATGTCTT TCCGGGTTGG ACTCAAGACG ATAGTTACCG 3541 GATAAGGCGC AGCGGTCGGA CTGAACGGGG GGTTCGTGCA TACAGTCCAG CTTGGAGCGA 3601 ACTGCCTACC CGGAACTGAG TGTCAGGCGT GGAATGAGAC AAACGCGGCC ATAACAGCGG 3661 AATGACACCG GTAAACCGAA AGGCAGGAAC AGGAGAGCGC ACGAGGGAGC CGCCAGGGGG 3721 AAACGCCTGG TATCTTTATA GTCCTGTCGG GTTTCGCCAC CACTGATTTG AGCGTCAGAT 3781 TTCGTGATGC TTGTCAGGGG GGCGGAGCCT ATGGAAAAAC GGCTTTGCCG CGGCCCTCTC 3841 ACTTCCCTGT TAAGTATCTT CCTGGCATCT TCCAGGAAAT CTCCGCCCCG TTCGTAAGCC 3901 ATTTCCGCTC GCCGCAGTCG AACGACCGAG CGTAGCGAGT CAGTGAGCGA GGAAGCGGAA 3961 TATATCCTGT ATCACATATT CTGCTGACGC ACCGGTGCAG CCTTTTTTCT CCTGCCACAT 4021 GAAGCACTTC ACTGACACCC TCATCAGTGC CAACATAGTA AGCCAGTATA CACTCCGCTA 4081 GCGCCCTGCT CGGATCTGTT GGACCGGACA GTAGTCATGG TTGATGGGCT GCCTGTATCG 4141 AGTGGTGATT TTGTGCCGAG CTGCCGGTCG GGGAGCTGTT GGCTGGCTGG TGGCAGGATA 4201 TATTGTGGTG TAAACAAATT GACGCTTAGA CAACTTAATA ACACATTGCG GACGTTTTTA 4261 ATGTACTGGG GTTGAACACT CTGTGGTCTC ATGCCGAATT CGGATCCGGA GGGGATACCA 4321 GAAACAAAAA AAGGGGAGCG GTTTCCCGCT CCCCTTCAAT AATTGGGCGC TTATGACAAC 4381 TTGACGGCTA CATCATTCAC TTTTTCTTCA CAACCGGCAC GGAACTCGCT CGGGCTGGCC 4441 CCGGTGCATT TTTTAAATAC CCGCGAGAAA TAGAGTTGAT CGTCAAAACC AACATTGCGA 4501 CCGACGGTGG CGATAGGCAT CCGGGTGGTG CTCAAAAGCA GCTTCGCCTG GCTGATACGT 4561 TGGTCCTCGC GCCAGCTTAA GACGCTAATC CCTAACTGCT GGCGGAAAAG ATGTGACAGA 4621 CGCGACGGCG ACAAGCAAAC ATGCTGTGCG ACGCTGGCGA TATCAAAATT GCTGTCTGCC 4681 AGGTGATCGC TGATGTACTG ACAAGCCTCG CGTACCCGAT TATCCATCGG TGGATGGAGC 4741 GACTCGTTAA TCGCTTCCAT GCGCCGCAGT AACAATTGCT CAAGCAGATT TATCGCCAGC 4801 AGCTCCGAAT AGCGCCCTTC CCCTTGCCCG GCGTTAATGA TTTGCCCAAA CAGGTCGCTG 4861 AAATGCGGCT GGTGCGCTTC ATCCGGGCGA AAGAACCCCG TATTGGCAAA TATTGACGGC 4921 CAGTTAAGCC ATTCATGCCA GTAGGCGCGC GGACGAAAGT AAACCCACTG GTGATACCAT 4981 TCGCGAGCCT CCGGATGACG ACCGTAGTGA TGAATCTCTC CTGGCGGGAA CAGCAAAATA 5041 TCACCCGGTC GGCAAACAAA TTCTCGTCCC TGATTTTTCA CCACCCCCTG ACCGCGAATG 5101 GTGAGATTGA GAATATAACC TTTCATTCCC AGCGGTCGGT CGATAAAAAA ATCGAGATAA 5161 CCGTTGGCCT CAATCGGCGT TAAACCCGCC ACCAGATGGG CATTAAACGA GTATCCCGGC 5221 AGCAGGGGAT CATTTTGCGC TTCAGCCATA CTTTTCATAC TCCCGCCATT CAGAGAAGAA 5281 ACCAATTGTC CATATTGCAT CAGACATTGC CGTCACTGCG TCTTTTACTG GCTCTTCTCG 5341 CTAACCAAAC CGGTAACCCC GCTTATTAAA AGCATTCTGT AACAAAGCGG GACCAAAGCC 5401 ATGACAAAAA CGCGTAACAA AAGTGTCTAT AATCACGGCA GAAAAGTCCA CATTGATTAT 5461 TTGCACGGCG TCACACTTTG CTATGCCATA GCATTTTTAT CCATAAGATT AGCGGATCCT 5521 ACCTGACGCT TTTTATCGCA ACTCTCTACT GTTTCTCCAT ACCCGTTTTT TGGGCTACTA 5581 AAAATAAGGA GGAAAAAAAA ATGACTAAAA AAATTTCATT CATTATTAAC GGCCAGGTTG 5641 AAATCTTTCC CGAAAGTGAT GATTTAGTGC AATCCATTAA TTTTGGTGAT AATAGTGTTT 5701 ACCTGCCAAT ATTGAATGAC TCTCATGTAA AAAACATTAT TGATTGTAAT GGAAATAACG 5761 AATTACGGTT GCATAACATT GTCAATTTTC TCTATACGGT AGGGCAAAGA TGGAAAAATG 5821 AAGAATACTC AAGACGCAGG ACATACATTC GTGACTTAAA AAAATATATG GGATATTCAG 5881 AAGAAATGGC TAAGCTAGAG GCCAATTGGA TATCTATGAT TTTATGTTCT AAAGGCGGCC 5941 TTTATGATGT TGTAGAAAAT GAACTTGGTT CTCGCCATAT CATGGATGAA TGGCTACCTC 6001 AGGATGAAAG TTATGTTCGG GCTTTTCCGA AAGGTAAATC TGTACATCTG TTGGCAGGTA 6061 ATGTTCCATT ATCTGGGATC ATGTCTATAT TACGCGCAAT TTTAACTAAG AATCAGTGTA 6121 TTATAAAAAC ATCGTCAACC GATCCTTTTA CCGCTAATGC ATTAGCGTTA AGTTTTATTG 
6181 ATGTAGACCC TAATCATCCG ATAACGCGCT CTTTATCTGT TATATATTGG CCCCACCAAG 6241 GTGATACATC ACTCGCAAAA GAAATTATGC GACATGCGGA TGTTATTGTC GCTTGGGGAG 6301 GGCCAGATGC GATTAATTGG GCGGTAGAGC ACGCGCCATC TTATGCTGAT GTGATTAAAT 6361 TTGGTTCTAA AAAGAGTCTT TGCATTATCG ATAATCCTGT TGATTTGACG TCCGCAGCGA 6421 CAGGTGCGGC TCATGATGTT TGTTTTTACG ATCAGCGAGC TTGTTTTTCT GCCCAAAACA 6481 TATATTACAT GGGAAATCAT TATGAGGAAT TTAAGTTAGC GTTGATAGAA AAACTTAATC 6541 TATATGCGCA TATATTACCG AATGCCAAAA AAGATTTTGA TGAAAAGGCG GCCTATTCTT 6601 TAGTTCAAAA AGAAAGCCTG TTTGCTGGAT TAAAAGTAGA GGTGGATATT CATCAACGTT 6661 GGATGATTAT TGAGTCAAAT GCAGGTGTGG AATTTAATCA ACCACTTGGC AGATGTGTGT 6721 ACCTTCATCA CGTCGATAAT ATTGAGCAAA TATTGCCTTA TGTTCAAAAA AATAAGACGC 6781 AAACCATATC TATTTTTCCT TGGGAGTCAT CATTTAAATA TCGAGATGCG TTAGCATTAA 6841 AAGGTGCGGA AAGGATTGTA GAAGCAGGAA TGAATAACAT ATTTCGAGTT GGTGGATCTC 6901 ATGACGGAAT GAGGCCGTTG CAACGATTAG TGACATATAT TTCTCATGAA AGGCCATCTA 6961 ACTATACGGC TAAGGATGTT GCGGTTGAAA TAGAACAGAC TCGATTCCTG GAAGAAGATA 7021 AGTTCCTTGT ATTTGTCCCA TAATAGGTAA AAGTATGGAA AATGAATCAA AATATAAAAC 7081 CATCGACCAC GTTATTTGTG TTGAAGGAAA TAAAAAAATT CATGTTTGGG AAACGCTGCC 7141 AGAAGAAAAC AGCCCAAAGA GAAAGAATGC CATTATTATT GCGTCTGGTT TTGCCCGCAG 7201 GATGGATCAT TTTGCTGGTC TGGCGGAATA TTTATCGCGG AATGGATTTC ATGTGATCCG 7261 CTATGATTCG CTTCACCACG TTGGATTGAG TTCAGGGACA ATTGATGAAT TTACAATGTC 7321 TATAGGAAAG CAGAGCTTGT TAGCAGTGGT TGATTGGTTA ACTACACGAA AAATAAATAA 7381 CTTCGGTATG TTGGCTTCCA GCTTATCTGC GCGGATAGCT TATGCAAGCC TATCTGAAAT 7441 CAATGCTTCG TTTTTAATCA CCGCAGTCGG TGTTGTTAAC TTAAGATATT CTCTTGAAAG 7501 AGCTTTAGGG TTTGATTATC TCAGTCTACC CATTAATGAA TTGCCGGATA ATCTGGATTT 7561 TGAAGGCCAT AAATTGGGTG CTGAAGTCTT TGCGAGAGAT TGTCTTGATT TTGGTTGGGA 7621 AGATTTAGCT TCTACAATTA ATAACATGAT GTATCTTGAT ATACCGTTTA TTGCTTTTAC 7681 TGCAAATAAC GATAATTGGG TCAAGCAAGA TGAAGTTATC ACATTGTTAT CAAATATTCG 7741 TAGTAATCGA TGCAAGATAT ATTCTTTGTT AGGAAGTTCG CATGACTTGA GTGAAAATTT 7801 AGTGGTCCTG CGCAATTTTT ATCAATCGGT TACGAAAGCC GCTATCGCGA TGGATAATGA 7861 TCATCTGGAT ATTGATGTTG ATATTACTGA ACCGTCATTT GAACATTTAA CTATTGCGAC 7921 AGTCAATGAA CGCCGAATGA GAATTGAGAT TGAAAATCAA GCAATTTCTC TGTCTTAAAA 7981 TCTATTGAGA TATTCTATCA CTCAAATAGC AATATAAGGA CTCTCTATGA AATTTGGAAA 8041 CTTTTTGCTT ACATACCAAC CTCCCCAATT TTCTCAAACA GAGGTAATGA AACGTTTGGT 8101 TAAATTAGGT CGCATCTCTG AGGAGTGTGG TTTTGATACC GTATGGTTAC TGGAGCATCA 8161 TTTCACGGAG TTTGGTTTGC TTGGTAACCC TTATGTCGCT GCTGCATATT TACTTGGCGC 8221 GACTAAAAAA TTGAATGTAG GAACTGCCGC TATTGTTCTT CCCACAGCCC ATCCAGTACG 8281 CCAACTTGAA GATGTGAATT TATTGGATCA AATGTCAAAA GGACGATTTC GGTTTGGTAT 8341 TTGCCGAGGG CTTTACAACA AGGACTTTCG CGTATTCGGC ACAGATATGA ATAACAGTCG 8401 CGCCTTAGCG GAATGCTGGT ACGGGCTGAT AAAGAATGGC ATGACAGAGG GATATATGGA 8461 AGCTGATAAT GAACATATCA AGTTCCATAA GGTAAAAGTA AACCCCGCGG CGTATAGCAG 8521 AGGTGGCGCA CCGGTTTATG TGGTGGCTGA ATCAGCTTCG ACGACTGAGT GGGCTGCTCA 8581 ATTTGGCCTA CCGATGATAT TAAGTTGGAT TATAAATACT AACGAAAAGA AAGCACAACT 8641 TGAGCTTTAT AATGAAGTGG CTCAAGAATA TGGGCACGAT ATTCATAATA TCGACCATTG 8701 CTTATCATAT ATAACATCTG TAGATCATGA CTCAATTAAA GCGAAAGAGA TTTGCCGGAA 8761 ATTTCTGGGG CATTGGTATG ATTCTTATGT GAATGCTACG ACTATTTTTG ATGATTCAGA 8821 CCAAACAAGA GGTTATGATT TCAATAAAGG GCAGTGGCGT GACTTTGTAT TAAAAGGACA 8881 TAAAGATACT AATCGCCGTA TTGATTACAG TTACGAAATC AATCCCGTGG GAACGCCGCA 8941 GGAATGTATT GACATAATTC AAAAAGACAT TGATGCTACA GGAATATCAA ATATTTGTTG 9001 TGGATTTGAA GCTAATGGAA CAGTAGACGA AATTATTGCT TCCATGAAGC TCTTCCAGTC 9061 TGATGTCATG CCATTTCTTA AAGAAAAACA ACGTTCGCTA TTATATTAGC TAAGGAGAAA 9121 GAAATGAAAT TTGGATTGTT CTTCCTTAAC TTCATCAATT CAACAACTGT TCAAGAACAA 
9181 AGTATAGTTC GTATGCAGGA AATAACGGAG TATGTTGATA AGTTGAATTT TGAACAGATT 9241 TTAGTGTATG AAAATCATTT TTCAGATAAT GGTGTTGTCG GCGCTCCTCT GACTGTTTCT 9301 GGTTTTCTGC TCGGTTTAAC AGAGAAAATT AAAATTGGTT CATTAAATCA CATCATTACA 9361 ACTCATCATC CTGTCGCCAT AGCGGAGGAA GCGTGCTTAT TGGATCAGTT AAGTGAAGGG 9421 AGATTTATTT TAGGGTTTAG TGATTGCGAA AAAAAAGATG AAATGCATTT TTTTAATCGC 9481 CCGGTTGAAT ATCAACAGCA ACTATTTGAA GAGTGTTATG AAATCATTAA CGATGCTTTA 9541 ACAACAGGCT ATTGTAATCC AGATAACGAT TTTTATAGCT TCCCTAAAAT ATCTGTAAAT 9601 CCCCATGCTT ATACGCCAGG CGGACCTCGG AAATATGTAA CAGCAACCAG TCATCATATT 9661 GTTGAGTGGG CGGCCAAAAA AGGTATTCCT CTCATCTTTA AGTGGGATGA TTCTAATGAT 9721 GTTAGATATG AATATGCTGA AAGATATAAA GCCGTTGCGG ATAAATATGA CGTTGACCTA 9781 TCAGAGATAG ACCATCAGTT AATGATATTA GTTAACTATA ACGAAGATAG TAATAAAGCT 9841 AAACAAGAGA CGCGTGCATT TATTAGTGAT TATGTTCTTG AAATGCACCC TAATGAAAAT 9901 TTCGAAAATA AACTTGAAGA AATAATTGCA GAAAACGCTG TCGGAAATTA TACGGAGTGT

9961 ATAACTGCGG CTAAGTTGGC AATTGAAAAG TGTGGTGCGA AAAGTGTATT GCTGTCCTTT 10021 GAACCAATGA ATGATTTGAT GAGCCAAAAA AATGTAATCA ATATTGTTGA TGATAATATT 10081 AAGAAGTACC ACATGGAATA TACCTAATAG ATTTCGAGTT GCAGCGAGGC GGCAAGTGAA 10141 CGAATCCCCA GGAGCATAGA TAACTATGTG ACTGGGGTGA GTGAAAGCAG CCAACAAAGC 10201 AGCAGCTTGA AAGATGAAGG GTATAAAAGA GTATGACAGC AGTGCTGCCA TACTTTCTAA 10261 TATTATCTTG AGGAGTAAAA CAGGTATGAC TTCATATGTT GATAAACAAG AAATTACAGC 10321 AAGCTCAGAA ATTGATGATT TGATTTTTTC GAGCGATCCA TTAGTGTGGT CTTACGACGA 10381 GCAGGAAAAA ATCAGAAAGA AACTTGTGCT TGATGCATTT CGTAATCATT ATAAACATTG 10441 TCGAGAATAT CGTCACTACT GTCAGGCACA CAAAGTAGAT GACAATATTA CGGAAATTGA 10501 TGACATACCT GTATTCCCAA CATCGGTTTT TAAGTTTACT CGCTTATTAA CTTCTCAGGA 10561 AAACGAGATT GAAAGTTGGT TTACCAGTAG CGGCACGAAT GGTTTAAAAA GTCAGGTGGC 10621 GCGTGACAGA TTAAGTATTG AGAGACTCTT AGGCTCTGTG AGTTATGGCA TGAAATATGT 10681 TGGTAGTTGG TTTGATCATC AAATAGAATT AGTCAATTTG GGACCAGATA GATTTAATGC 10741 TCATAATATT TGGTTTAAAT ATGTTATGAG TTTGGTGGAA TTGTTATATC CTACGACATT 10801 TACCGTAACA GAAGAACGAA TAGATTTTGT TAAAACATTG AATAGTCTTG AACGAATAAA 10861 AAATCAAGGG AAAGATCTTT GTCTTATTGG TTCGCCATAC TTTATTTATT TACTCTGCCA 10921 TTATATGAAA GATAAAAAAA TCTCATTTTC TGGAGATAAA AGCCTTTATA TCATAACCGG 10981 AGGCGGCTGG AAAAGTTACG AAAAAGAATC TCTGAAACGT GATGATTTCA ATCATCTTTT 11041 ATTTGATACT TTCAATCTCA GTGATATTAG TCAGATCCGA GATATATTTA ATCAAGTTGA 11101 ACTCAACACT TGTTTCTTTG AGGATGAAAT GCAGCGTAAA CATGTTCCGC CGTGGGTATA 11161 TGCGCGAGCG CTTGATCCTG AAACGTTGAA ACCTGTACCT GATGGAACGC CGGGGTTGAT 11221 GAGTTATATG GATGCGTCAG CAACCAGTTA TCCAGCATTT ATTGTTACCG ATGATGTCGG 11281 GATAATTAGC AGAGAATATG GTAAGTATCC CGGCGTGCTC GTTGAAATTT TACGTCGCGT 11341 CAATACGAGG ACGCAGAAAG GGTGTGCTTT GAGCTTAACC GAAGCGTTTG ATAGTTGAGC 11401 TTCTCGGTAC CAAATTCCAG AAAAGAGGCC TCCCGAAAGG GGGGCCTTTT TTCGTTTTGG 11461 TCCCGCTGCA ATGAGACCGA GGATGCACAT GTGACCGAGG GACACGAA 


\section{DNA sequence of pSV012}

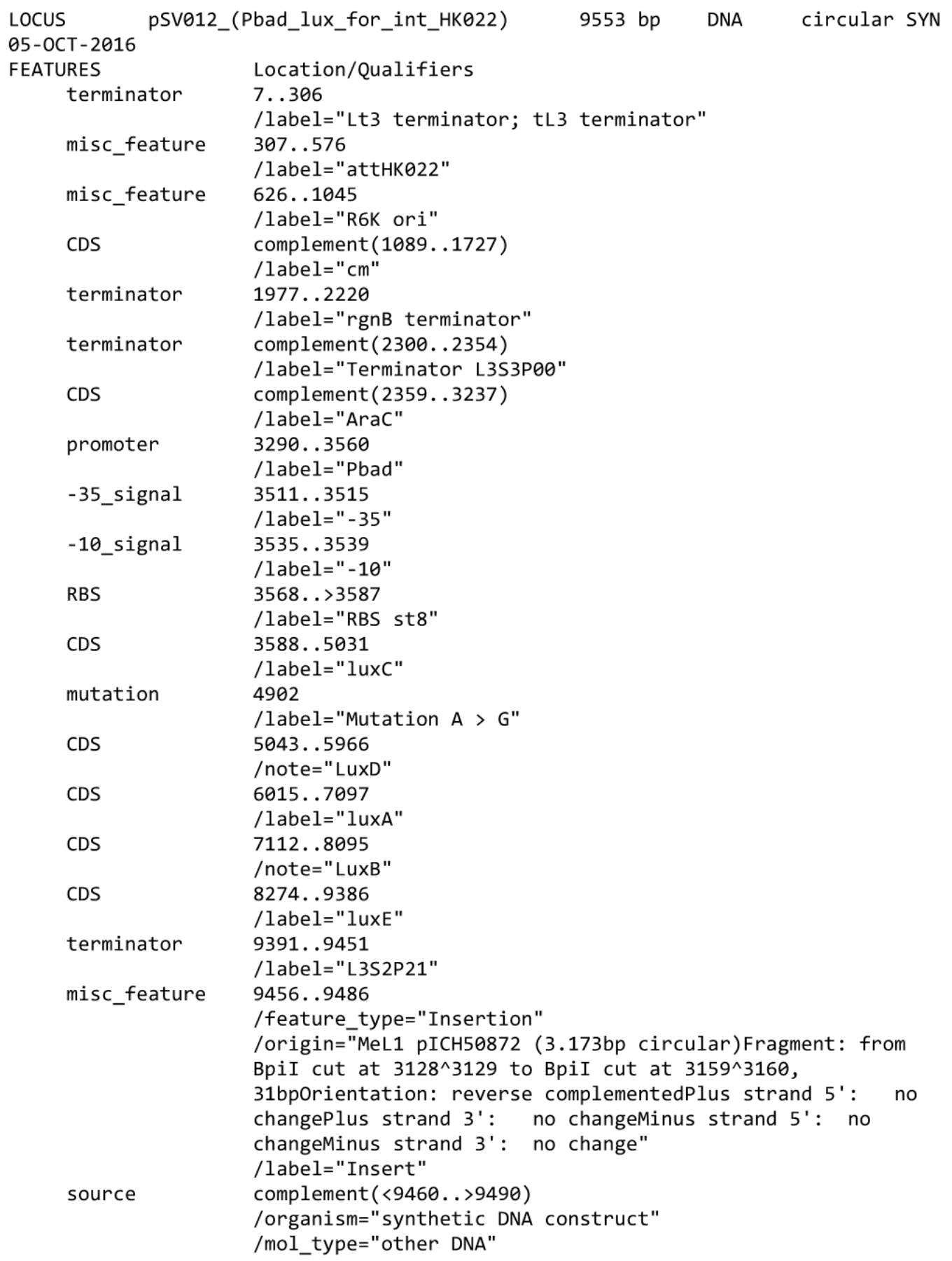


ORIGIN

1 TCATGTTTGA CAGCTTATCA CTGATCAGTG AATTAATGGC GATGACGCAT CCTCACGATA 61 ATATCCGGGT AGGCGCAATC ACTTTCGTCT CTACTCCGTT ACAAAGCGAG GCTGGGTATT 121 TCCCGGCCTT TCTGTTATCC GAAATCCACT GAAAGCACAG CGGCTGGCTG AGGAGATAAA 181 TAATAAACGA GGGGCTGTAT GCACAAAGCA TCTTCTGTTG AGTTAAGAAC GAGTATCGAG 241 ATGGCACATA GCCTTGCTCA AATTGGAATC AGGTTTGTGC CAATACCAGT AGAAACAGAC 301 GAAGAAGCTA GCTAATGCTC TGTCTCAGGT CACTAATACT ATCTAAGTAG TTGATTCATA 361 GTGACTGGAT ATGTTGCGTT TTGTCGCATT ATGTAGTCTA TCATTTAACC ACAGATTAGT 421 GTAATGCGAT GATTTTTAAG TGATTAATGT TATTTTGTCA TCCTTTAGGT GAATAAGTTG 481 TATATTTAAA ATCTCTTTAA TTATCAGTAA ATTAATGTAA GTAGGTCATT ATTAGTCAAA 541 ATAAAATCAT TTGTCGATTT CAATTTTGTC CCATGGCAGT TCCTATTCCG AAGTTCCTAT 601 TCTCTAGAAA GTATAGGAAC TTCTAATTCC CATGTCAGCC GTTAAGTGTT CCTGTGTCAC 661 TCAAAATTGC TTTGAGAGGC TCTAAGGGCT TCTCAGTGCG TTACATCCCT GGCTTGTTGT 721 CCACAACCGT TAAACCTTAA AAGCTTTAAA AGCCTTATAT ATTCTTTTTT TTCTTATAAA 781 ACTTAAAACC TTAGAGGCTA TTTAAGTTGC TGATTTATAT TAATTTTATT GTTCAAACAT 841 GAGAGCTTAG TACGTGAAAC ATGAGAGCTT AGTACGTTAG CCATGAGAGC TTAGTACGTT 901 AGCCATGAGG GTTTAGTTCG TTAAACATGA GAGCTTAGTA CGTTAAACAT GAGAGCTTAG 961 TACGTGAAAC ATGAGAGCTT AGTACGTACT ATCAACAGGT TGAACTGCTG ATCTTCAGAT 1021 CCTCTACGCC GGACGCATCG TGGCCGGATC TTGATTTAAA TGGCGCGCCT TACGCCCCGC 1081 CCTGCCACTC ATCGCAGTAC TGTTGTATTC ATTAAGCATC TGCCGACATG GAAGCCATCA 1141 CAAACGGCAT GATGAACCTG AATCGCCAGC GGCATCAGCA CCTTGTCGCC TTGCGTATAA 1201 TATTTGCCCA TGGTGAAAAC GGGGGCGAAG AAGTTGTCCA TATTGGCCAC GTTTAAATCA 1261 AAACTGGTGA AACTCACCCA GGGATTGGCT GAGACGAAAA ACATATTCTC AATAAACCCT 1321 TTAGGGAAAT AGGCCAGGTT TTCACCGTAA CACGCCACAT CTTGCGAATA TATGTGTAGA 1381 AACTGCCGGA AATCGTCGTG GTATTCACTC CAGAGCGATG AAAACGTTTC AGTTTGCTCA 1441 TGGAAAACGG TGTAACAAGG GTGAACACTA TCCCATATCA CCAGCTCACC GTCTTTCATT 1501 GCCATACGTA ATTCCGGATG AGCATTCATC AGGCGGGCAA GAATGTGAAT AAAGGCCGGA 1561 TAAAACTTGT GCTTATTTTT CTTTACGGTC TTTAAAAAGG CCGTAATATC CAGCTGAACG 1621 GTCTGGTTAT AGGTACATTG AGCAACTGAC TGAAATGCCT CAAAATGTTC TTTACGATGC 1681 CATTGGGATA TATCAACGGT GGTATATCCA GTGATTTTTT TCTCCATTTT AGCTTCCTTA 1741 GCTCCTGAAA ATCTCGACAA CTCAAAAAAT ACGCCCGGTA GTGATCTTAT TTCATTATGG 1801 TGAAAGTTGG AACCTCTTAC GTGCCGATCA ACGTCTCATT TTCGCCAAAA GTTGGCCCAG 1861 GGCTTCCCGG TATCAACAGG GACACCAGGA TTTATTTATT CTGCGAAGTG ATCTTCCGTC 1921 ACAGGTAGGA AGTTCCTATT CCGAAGTTCC TATTCTCTAG AAAGTATAGG AACTTCGTCT 1981 CCCCATGCGA GAGTAGGGAA CTGCCAGGCA TCAAATAAAA CGAAAGGCTC AGTCGAAAGA 2041 CTGGGCCTTT CGTTTTATCT GTTGTTTGTC GGTGAACGCT CTCCTGAGTA GGACAAATCC 2101 GCCGGGAGCG GATTTGAACG TTGCGAAGCA ACGGCCCGGA GGGTGGCGGG CAGGACGCCC 2161 GCCATAAACT GCCAGGCATC AAATTAAGCA GAAGGCCATC CTGACGGATG GCCTTTTTGC 2221 GTGGCCAGTC ACATTGCGGA CGTTTTTAAT GTACTGGGGT TGAACACTCT GTGGTCTCAT 2281 GCCGAATTCG GATCCGGAGG GGATACCAGA AACAAAAAAA GGGGAGCGGT TTCCCGCTCC 2341 CCTTCAATAA TTGGGCGCTT ATGACAACTT GACGGCTACA TCATTCACTT TTTCTTCACA 2401 ACCGGCACGG AACTCGCTCG GGCTGGCCCC GGTGCATTTT TTAAATACCC GCGAGAAATA 2461 GAGTTGATCG TCAAAACCAA CATTGCGACC GACGGTGGCG ATAGGCATCC GGGTGGTGCT 2521 CAAAAGCAGC TTCGCCTGGC TGATACGTTG GTCCTCGCGC CAGCTTAAGA CGCTAATCCC 2581 TAACTGCTGG CGGAAAAGAT GTGACAGACG CGACGGCGAC AAGCAAACAT GCTGTGCGAC 2641 GCTGGCGATA TCAAAATTGC TGTCTGCCAG GTGATCGCTG ATGTACTGAC AAGCCTCGCG 2701 TACCCGATTA TCCATCGGTG GATGGAGCGA CTCGTTAATC GCTTCCATGC GCCGCAGTAA 2761 CAATTGCTCA AGCAGATTTA TCGCCAGCAG CTCCGAATAG CGCCCTTCCC CTTGCCCGGC 2821 GTTAATGATT TGCCCAAACA GGTCGCTGAA ATGCGGCTGG TGCGCTTCAT CCGGGCGAAA 2881 GAACCCCGTA TTGGCAAATA TTGACGGCCA GTTAAGCCAT TCATGCCAGT AGGCGCGCGG 
2941 ACGAAAGTAA ACCCACTGGT GATACCATTC GCGAGCCTCC GGATGACGAC CGTAGTGATG 3001 AATCTCTCCT GGCGGGAACA GCAAAATATC ACCCGGTCGG CAAACAAATT CTCGTCCCTG 3061 ATTTTTCACC ACCCCCTGAC CGCGAATGGT GAGATTGAGA ATATAACCTT TCATTCCCAG 3121 CGGTCGGTCG ATAAAAAAAT CGAGATAACC GTTGGCCTCA ATCGGCGTTA AACCCGCCAC 3181 CAGATGGGCA TTAAACGAGT ATCCCGGCAG CAGGGGATCA TTTTGCGCTT CAGCCATACT 3241 TTTCATACTC CCGCCATTCA GAGAAGAAAC CAATTGTCCA TATTGCATCA GACATTGCCG 3301 TCACTGCGTC TTTTACTGGC TCTTCTCGCT AACCAAACCG GTAACCCCGC TTATTAAAAG 3361 CATTCTGTAA CAAAGCGGGA CCAAAGCCAT GACAAAAACG CGTAACAAAA GTGTCTATAA 3421 TCACGGCAGA AAAGTCCACA TTGATTATTT GCACGGCGTC ACACTTTGCT ATGCCATAGC 3481 ATTTTTATCC ATAAGATTAG CGGATCCTAC CTGACGCTTT TTATCGCAAC TCTCTACTGT 3541 TTCTCCATAC CCGTTTTTTG GGCTACTAAA AATAAGGAGG AAAAAAAAAT GACTAAAAAA 3601 ATTTCATTCA TTATTAACGG CCAGGTTGAA ATCTTTCCCG AAAGTGATGA TTTAGTGCAA 3661 TCCATTAATT TTGGTGATAA TAGTGTTTAC CTGCCAATAT TGAATGACTC TCATGTAAAA 3721 AACATTATTG ATTGTAATGG AAATAACGAA TTACGGTTGC ATAACATTGT CAATTTTCTC 3781 TATACGGTAG GGCAAAGATG GAAAAATGAA GAATACTCAA GACGCAGGAC ATACATTCGT 3841 GACTTAAAAA AATATATGGG ATATTCAGAA GAAATGGCTA AGCTAGAGGC CAATTGGATA 3901 TCTATGATTT TATGTTCTAA AGGCGGCCTT TATGATGTTG TAGAAAATGA ACTTGGTTCT 3961 CGCCATATCA TGGATGAATG GCTACCTCAG GATGAAAGTT ATGTTCGGGC TTTTCCGAAA 4021 GGTAAATCTG TACATCTGTT GGCAGGTAAT GTTCCATTAT CTGGGATCAT GTCTATATTA 4081 CGCGCAATTT TAACTAAGAA TCAGTGTATT ATAAAAACAT CGTCAACCGA TCCTTTTACC 4141 GCTAATGCAT TAGCGTTAAG TTTTATTGAT GTAGACCCTA ATCATCCGAT AACGCGCTCT 4201 TTATCTGTTA TATATTGGCC CCACCAAGGT GATACATCAC TCGCAAAAGA AATTATGCGA 4261 CATGCGGATG TTATTGTCGC TTGGGGAGGG CCAGATGCGA TTAATTGGGC GGTAGAGCAC 4321 GCGCCATCTT ATGCTGATGT GATTAAATTT GGTTCTAAAA AGAGTCTTTG CATTATCGAT 4381 AATCCTGTTG ATTTGACGTC CGCAGCGACA GGTGCGGCTC ATGATGTTTG TTTTTACGAT 4441 CAGCGAGCTT GTTTTTCTGC CCAAAACATA TATTACATGG GAAATCATTA TGAGGAATTT 4501 AAGTTAGCGT TGATAGAAAA ACTTAATCTA TATGCGCATA TATTACCGAA TGCCAAAAAA 4561 GATTTTGATG AAAAGGCGGC CTATTCTTTA GTTCAAAAAG AAAGCCTGTT TGCTGGATTA 4621 AAAGTAGAGG TGGATATTCA TCAACGTTGG ATGATTATTG AGTCAAATGC AGGTGTGGAA 4681 TTTAATCAAC CACTTGGCAG ATGTGTGTAC CTTCATCACG TCGATAATAT TGAGCAAATA 4741 TTGCCTTATG TTCAAAAAAA TAAGACGCAA ACCATATCTA TTTTTCCTTG GGAGTCATCA 4801 TTTAAATATC GAGATGCGTT AGCATTAAAA GGTGCGGAAA GGATTGTAGA AGCAGGAATG 4861 AATAACATAT TTCGAGTTGG TGGATCTCAT GACGGAATGA GGCCGTTGCA ACGATTAGTG 4921 ACATATATTT CTCATGAAAG GCCATCTAAC TATACGGCTA AGGATGTTGC GGTTGAAATA 4981 GAACAGACTC GATTCCTGGA AGAAGATAAG TTCCTTGTAT TTGTCCCATA ATAGGTAAAA 5041 GTATGGAAAA TGAATCAAAA TATAAAACCA TCGACCACGT TATTTGTGTT GAAGGAAATA 5101 AAAAAATTCA TGTTTGGGAA ACGCTGCCAG AAGAAAACAG CCCAAAGAGA AAGAATGCCA 5161 TTATTATTGC GTCTGGTTTT GCCCGCAGGA TGGATCATTT TGCTGGTCTG GCGGAATATT 5221 TATCGCGGAA TGGATTTCAT GTGATCCGCT ATGATTCGCT TCACCACGTT GGATTGAGTT 5281 CAGGGACAAT TGATGAATTT ACAATGTCTA TAGGAAAGCA GAGCTTGTTA GCAGTGGTTG 5341 ATTGGTTAAC TACACGAAAA ATAAATAACT TCGGTATGTT GGCTTCCAGC TTATCTGCGC 5401 GGATAGCTTA TGCAAGCCTA TCTGAAATCA ATGCTTCGTT TTTAATCACC GCAGTCGGTG 5461 TTGTTAACTT AAGATATTCT CTTGAAAGAG CTTTAGGGTT TGATTATCTC AGTCTACCCA 5521 TTAATGAATT GCCGGATAAT CTGGATTTTG AAGGCCATAA ATTGGGTGCT GAAGTCTTTG 5581 CGAGAGATTG TCTTGATTTT GGTTGGGAAG ATTTAGCTTC TACAATTAAT AACATGATGT 5641 ATCTTGATAT ACCGTTTATT GCTTTTACTG CAAATAACGA TAATTGGGTC AAGCAAGATG 5701 AAGTTATCAC ATTGTTATCA AATATTCGTA GTAATCGATG CAAGATATAT TCTTTGTTAG 5761 GAAGTTCGCA TGACTTGAGT GAAAATTTAG TGGTCCTGCG CAATTTTTAT CAATCGGTTA 5821 CGAAAGCCGC TATCGCGATG GATAATGATC ATCTGGATAT TGATGTTGAT ATTACTGAAC 5881 CGTCATTTGA ACATTTAACT ATTGCGACAG TCAATGAACG CCGAATGAGA ATTGAGATTG 
5941 AAAATCAAGC AATTTCTCTG TCTTAAAATC TATTGAGATA TTCTATCACT CAAATAGCAA 6001 TATAAGGACT CTCTATGAAA TTTGGAAACT TTTTGCTTAC ATACCAACCT CCCCAATTTT 6061 CTCAAACAGA GGTAATGAAA CGTTTGGTTA AATTAGGTCG CATCTCTGAG GAGTGTGGTT 6121 TTGATACCGT ATGGTTACTG GAGCATCATT TCACGGAGTT TGGTTTGCTT GGTAACCCTT 6181 ATGTCGCTGC TGCATATTTA CTTGGCGCGA CTAAAAAATT GAATGTAGGA ACTGCCGCTA 6241 TTGTTCTTCC CACAGCCCAT CCAGTACGCC AACTTGAAGA TGTGAATTTA TTGGATCAAA 6301 TGTCAAAAGG ACGATTTCGG TTTGGTATTT GCCGAGGGCT TTACAACAAG GACTTTCGCG 6361 TATTCGGCAC AGATATGAAT AACAGTCGCG CCTTAGCGGA ATGCTGGTAC GGGCTGATAA 6421 AGAATGGCAT GACAGAGGGA TATATGGAAG CTGATAATGA ACATATCAAG TTCCATAAGG 6481 TAAAAGTAAA CCCCGCGGCG TATAGCAGAG GTGGCGCACC GGTTTATGTG GTGGCTGAAT 6541 CAGCTTCGAC GACTGAGTGG GCTGCTCAAT TTGGCCTACC GATGATATTA AGTTGGATTA 6601 TAAATACTAA CGAAAAGAAA GCACAACTTG AGCTTTATAA TGAAGTGGCT CAAGAATATG 6661 GGCACGATAT TCATAATATC GACCATTGCT TATCATATAT AACATCTGTA GATCATGACT 6721 CAATTAAAGC GAAAGAGATT TGCCGGAAAT TTCTGGGGCA TTGGTATGAT TCTTATGTGA 6781 ATGCTACGAC TATTTTTGAT GATTCAGACC AAACAAGAGG TTATGATTTC AATAAAGGGC 6841 AGTGGCGTGA CTTTGTATTA AAAGGACATA AAGATACTAA TCGCCGTATT GATTACAGTT 6901 ACGAAATCAA TCCCGTGGGA ACGCCGCAGG AATGTATTGA CATAATTCAA AAAGACATTG 6961 ATGCTACAGG AATATCAAAT ATTTGTTGTG GATTTGAAGC TAATGGAACA GTAGACGAAA 7021 TTATTGCTTC CATGAAGCTC TTCCAGTCTG ATGTCATGCC ATTTCTTAAA GAAAAACAAC 7081 GTTCGCTATT ATATTAGCTA AGGAGAAAGA AATGAAATTT GGATTGTTCT TCCTTAACTT 7141 CATCAATTCA ACAACTGTTC AAGAACAAAG TATAGTTCGT ATGCAGGAAA TAACGGAGTA 7201 TGTTGATAAG TTGAATTTTG AACAGATTTT AGTGTATGAA AATCATTTTT CAGATAATGG 7261 TGTTGTCGGC GCTCCTCTGA CTGTTTCTGG TTTTCTGCTC GGTTTAACAG AGAAAATTAA 7321 AATTGGTTCA TTAAATCACA TCATTACAAC TCATCATCCT GTCGCCATAG CGGAGGAAGC 7381 GTGCTTATTG GATCAGTTAA GTGAAGGGAG ATTTATTTTA GGGTTTAGTG ATTGCGAAAA 7441 AAAAGATGAA ATGCATTTTT TTAATCGCCC GGTTGAATAT CAACAGCAAC TATTTGAAGA 7501 GTGTTATGAA ATCATTAACG ATGCTTTAAC AACAGGCTAT TGTAATCCAG ATAACGATTT 7561 TTATAGCTTC CCTAAAATAT CTGTAAATCC CCATGCTTAT ACGCCAGGCG GACCTCGGAA 7621 ATATGTAACA GCAACCAGTC ATCATATTGT TGAGTGGGCG GCCAAAAAAG GTATTCCTCT 7681 CATCTTTAAG TGGGATGATT CTAATGATGT TAGATATGAA TATGCTGAAA GATATAAAGC 7741 CGTTGCGGAT AAATATGACG TTGACCTATC AGAGATAGAC CATCAGTTAA TGATATTAGT 7801 TAACTATAAC GAAGATAGTA ATAAAGCTAA ACAAGAGACG CGTGCATTTA TTAGTGATTA 7861 TGTTCTTGAA ATGCACCCTA ATGAAAATTT CGAAAATAAA CTTGAAGAAA TAATTGCAGA 7921 AAACGCTGTC GGAAATTATA CGGAGTGTAT AACTGCGGCT AAGTTGGCAA TTGAAAAGTG 7981 TGGTGCGAAA AGTGTATTGC TGTCCTTTGA ACCAATGAAT GATTTGATGA GCCAAAAAAA 8041 TGTAATCAAT ATTGTTGATG ATAATATTAA GAAGTACCAC ATGGAATATA CCTAATAGAT 8101 TTCGAGTTGC AGCGAGGCGG CAAGTGAACG AATCCCCAGG AGCATAGATA ACTATGTGAC 8161 TGGGGTGAGT GAAAGCAGCC AACAAAGCAG CAGCTTGAAA GATGAAGGGT ATAAAAGAGT 8221 ATGACAGCAG TGCTGCCATA CTTTCTAATA TTATCTTGAG GAGTAAAACA GGTATGACTT 8281 CATATGTTGA TAAACAAGAA ATTACAGCAA GCTCAGAAAT TGATGATTTG ATTTTTTCGA 8341 GCGATCCATT AGTGTGGTCT TACGACGAGC AGGAAAAAAT CAGAAAGAAA CTTGTGCTTG 8401 ATGCATTTCG TAATCATTAT AAACATTGTC GAGAATATCG TCACTACTGT CAGGCACACA 8461 AAGTAGATGA CAATATTACG GAAATTGATG ACATACCTGT ATTCCCAACA TCGGTTTTTA 8521 AGTTTACTCG CTTATTAACT TCTCAGGAAA ACGAGATTGA AAGTTGGTTT ACCAGTAGCG 8581 GCACGAATGG TTTAAAAAGT CAGGTGGCGC GTGACAGATT AAGTATTGAG AGACTCTTAG 8641 GCTCTGTGAG TTATGGCATG AAATATGTTG GTAGTTGGTT TGATCATCAA ATAGAATTAG 8701 TCAATTTGGG ACCAGATAGA TTTAATGCTC ATAATATTTG GTTTAAATAT GTTATGAGTT 8761 TGGTGGAATT GTTATATCCT ACGACATTTA CCGTAACAGA AGAACGAATA GATTTTGTTA 8821 AAACATTGAA TAGTCTTGAA CGAATAAAAA ATCAAGGGAA AGATCTTTGT CTTATTGGTT 8881 CGCCATACTT TATTTATTTA CTCTGCCATT ATATGAAAGA TAAAAAAATC TCATTTTCTG 
8941 GAGATAAAAG CCTTTATATC ATAACCGGAG GCGGCTGGAA AAGTTACGAA AAAGAATCTC 9001 TGAAACGTGA TGATTTCAAT CATCTTTTAT TTGATACTTT CAATCTCAGT GATATTAGTC 9061 AGATCCGAGA TATATTTAAT CAAGTTGAAC TCAACACTTG TTTCTTTGAG GATGAAATGC 9121 AGCGTAAACA TGTTCCGCCG TGGGTATATG CGCGAGCGCT TGATCCTGAA ACGTTGAAAC 9181 CTGTACCTGA TGGAACGCCG GGGTTGATGA GTTATATGGA TGCGTCAGCA ACCAGTTATC 9241 CAGCATTTAT TGTTACCGAT GATGTCGGGA TAATTAGCAG AGAATATGGT AAGTATCCCG 9301 GCGTGCTCGT TGAAATTTTA CGTCGCGTCA ATACGAGGAC GCAGAAAGGG TGTGCTTTGA 9361 GCTTAACCGA AGCGTTTGAT AGTTGAGCTT CTCGGTACCA AATTCCAGAA AAGAGGCCTC 9421 CCGAAAGGGG GGCCTTTTTT CGTTTTGGTC CCGCTGCAAT GAGACCGAGG ATGCACATGT 9481 GACCGAGGGA CACGAAGTGA TCCGTTTAAA CTATCAGTGT TTGACAGGAT ATATTGGCGG 9541 GTAAACCTAA GAG 